\title{
Backgrounds from tensor models: A proposal
}

\author{
Pablo Diaz \\ Departamento de Matemática Aplicada, Universidad de Zaragoza, Zaragoza, 50009, Spain \\ and Centro de Astropartículas y Física de Altas Energas (CAPA), Zaragoza, 50009, Spain
}

(Received 29 October 2020; accepted 22 February 2021; published 17 March 2021)

\begin{abstract}
Although tensor models are serious candidates for a theory of quantum gravity, a connection with classical spacetimes has been elusive thus far. I aim to fill this gap by proposing a neat connection between tensor theory and Euclidean gravity at the classical level. The main departure from the usual approach is the use of Schur invariants (instead of monomial invariants) as manifold partners. Classical spacetime features can be identified naturally on the tensor side in this new setup. A notion of locality is shown to emerge through Ward identities, where proximity between spacetime points translates into vicinity between Young diagram corners.
\end{abstract}

DOI: 10.1103/PhysRevD.103.066010

\section{INTRODUCTION}

One of the most fascinating challenges of physics today is to understand the quantum nature of spacetime. In contrast to other theories which were developed in parallel with experiments, like quantum mechanics, the development of quantum gravity has to rely only on mathematical consistency due to the high energy experiments that would be required in order to test it in the lab. A necessary check for any quantum gravity theory is the recovery of Einstein gravity at the classical limit.

We still lack a unified framework for quantum gravity. However, several approaches have brought important insights from different perspectives. Known approaches to quantum gravity are string theory, noncommutative geometry, holography, spacetime triangulations, canonical quantum gravity, and tensor theories. One of these, if not a new option, will likely prevail in the future. It will probably be the one that offers a friendlier picture or permits more accurate calculations. As a comment, I must say that I do not find any conflict in the diversity of the current spectrum of (sometimes overlapping) theories. At the end of the day, the success of a physical model to make predictions relies mostly on the consideration of the relevant degrees of freedom for the given phenomenon, and mathematics is rich enough to allocate those into separate frameworks.

An exciting feature of tensor theories is the idea of "spacetime emergence". Spacetime is not assumed a priori,

*pablodiazbe@gmail.com

Published by the American Physical Society under the terms of the Creative Commons Attribution 4.0 International license. Further distribution of this work must maintain attribution to the author(s) and the published article's title, journal citation, and DOI. Funded by SCOAP. but it is expected to appear combinatorially. Tensor models are expected to give a discretized (combinatorial) description of the Euclidean quantum gravity partition function

$$
Z=\int d g e^{-I_{E}[g]}
$$

The usual approach to quantum gravity from tensor models, and the line I will follow in this paper, is precisely the discretization of Eq. (1.1). However, there are recent developments, with interesting results, where, by means of the connection between tensor and matrix theories, they apply holographic results and techniques to make contact with gravity; see, for instance, [1]. Perhaps these two strategies, tensors as tools for discretization and holographic tensors, are not so different. As an example, the $c=1$ string was motivated by summing over surfaces, but in the end there is an AdS/CFT-like duality between matrix quantum mechanics and noncritical strings.

The idea of describing spacetime by means of tensor models comes historically from the remarkable success of matrix theories in describing two-dimensional gravity [2] at the sector where the matrix size, $N$, is large. However, the first tensor models that were proposed [3-5] were pathological at large $N$, so the subject faded from discussion. In 2009, with the arrival of color tensor models [6,7], the situation changed. A well-defined $1 / N$ expansion was found [8-10], the subject was revitalized [11-22], and since then tensor models have become firm candidates for a theory of quantum gravity. The interested reader can find more comprehensive bibliographic information in [23] and the references therein.

A precise connection between tensor invariants and piecewise linear (PL) manifolds has been established. Invariants in tensor models are linear combinations of 
monomials made of $n$ copies of a tensor $T$ which are contracted with $n$ copies of its complex conjugate $\bar{T}$ in a certain way. There is a natural map between these monomials and PL manifolds: each pattern of contraction is interpreted as dictating how to glue simplices along their faces to build the manifold. Monomial invariants are, so to say, the skeletons of PL manifolds. Remarkably enough, providing suitable identifications, it has been proven that the dynamics of tensor models reproduces the dynamics of triangulations driven by the Regge calculus [24]. Specifically, the amplitude of each tensor invariant appearing as a Wick contraction in the computation of an expectation value is associated with the amplitude of the corresponding triangulation related to the Regge action. This result, together with the known fact that Regge discretizations lead to Einstein gravity at the continuum limit, establishes a solid connection between tensor models and gravity. Let us remember that this connection involves some gauge fixing on the gravity side since the triangulations must be equilateral.

Despite the success of the combinatorial description of PL manifolds, the classical limit and the description of backgrounds by tensor models has been elusive thus far. The purpose of this paper is to fill this gap. The main point of departure of the paper from the usual setup is the consideration of a different set of invariants as partners of classical backgrounds. Using representation theory arguments, a basis of invariants for any values of $n$ and $N$, the restricted Schur basis, has been found $[12,13,15,20]$. There is a prominent set of invariants, the Schur invariants, which are easily constructed from characters of the symmetric group and related to the restricted Schur basis by simple linear combinations. Schur invariants are the candidates I propose for background partners. I will justify this choice in Sec. III A by showing how, in the presence of a large Schur invariant, the three-point function factorizes, meaning that any density matrix describing multiparticle states turns diagonal.

Schur invariants are linear combinations of monomials weighed by characters. They are labeled by $d$ Young diagrams with $n$ boxes each and a maximum of $N$ rows. So, from the standard point of view (that is, with the identification of monomials with PL manifolds), classical backgrounds (Schur invariants) occur in our setup as a collective behavior of quantum contributions (monomial invariants).

The first problem we face with the new set of invariants is that we do not know at first how to relate them to manifolds. Remember that as we leave monomials we lose the prescription to identify patterns of contraction with simplicial tilings. This is a central question and marks the starting point of the paper. In order to establish a connection between Schur invariants and manifolds, I equate the partition functions of Euclidean gravity and tensor theory at a saddle point. This equation must be thought of as an ansatz. Using generic actions for both theories and some mathematical treatment, I obtain Eq. (4.14), which tells us that, at the classical level, the curvature of the (discrete) manifold at each point is given by the expectation value of a corresponding Schur invariant. Furthermore, for Einstein gravity, the expectation value must be computed with the free tensor action. Notice that the connection between tensor theory and gravity that we propose does not involve any gauge fixing.

A notion of locality arises in our setup via the Ward identities. Ward identities in tensor models involve the action of two operators, cut and join, defined in Eqs. (4.3) and (4.4), respectively. Locality is linked to the cut action, which hits on invariants as a derivative. That the action of the cut operator over Schur invariants produces all Schur invariants coming from the original with one box deleted in each label [see (E10)] is crucial for a notion of locality in tensor models. This enables us to map the corners of the $d$ Young diagrams of a given Schur invariant to a grid of "physical" discrete spacetime points in a way that nearby points in the grid are nearby corners in the Young diagrams.

Another insight we have from the use of Schur invariants in relation with classical backgrounds is that the limits $n, N \rightarrow \infty$ are not independent. This happens because the asymptotic Young diagrams must be limit shapes [25]; otherwise Schur invariants do not have a well-defined asymptotic limit. ${ }^{1}$ This fact, together with restriction of the number of rows in each diagram to at most $N$, tells us that $n$ must grow as $N^{2}$. One could wonder about the role (if any) that the melonic sector will have in this picture. The answer is that for large invariants of size $n \sim N^{2}$ the melonic contribution to any expectation value is negligible. ${ }^{2}$ Thus, melon invariants, although leading for short invariants, play no role in our proposal for classical backgrounds. ${ }^{3}$ Physical quantities in general relativity should have a tensorial counterpart in the appropriate limit. If we think of asymptotically flat spacetimes, for simplicity, the Arnowitt-DeserMisner (ADM) mass is a charge associated with the whole geometry. But what is the ADM mass in the tensor world? Using general arguments, I claim that the ADM mass must be proportional to $n / N^{2}$, which is a fixed quantity for a given Schur invariant and remains finite at $n, N \rightarrow \infty$.

The paper is organized as follows. Section II provides a brief introduction to tensor models: invariants, the restricted

\footnotetext{
${ }^{1}$ This is related to inductive definition and the representation theory of $S_{\infty}$. See the classical results by Thoma in [26] or a didactic review by Okounkov [27].

${ }^{2}$ Here the same discussion is applied as in [28] for invariants with $n \sim N$ (parallel to the original arguments in tensor models in [29]), where it was shown that for such large invariants the contribution of nonmelonic diagrams, being so numerous, overwhelms the entire sum.

${ }^{3}$ Along the same lines, see [1], where, using holographic arguments, it is claimed that the melonic sector does not lead to an emergent geometry.
} 
Schur basis, Schur invariants, the action, the partition function, and Gaussian correlators. It also sets the notation that I will be using later. In Sec. III, I start by verifying the correspondence between large Schur invariants and classical backgrounds. I show how, in the presence of a large Schur invariant, three-point functions factorize. This fact is interpreted as the large Schur invariant behaving as a classical background, where multiparticle states are seen as independent excitations. Afterward, in Sec. III B, I put into contact both theories, tensor and gravity, by equating the respective partition functions at the saddle point in Eq. (3.31). The main result of the section is Eq. (3.40), where the on-shell Hilbert-Einstein action is computed using the Gaussian correlator of the corresponding tensor invariant. Locality is tackled in Sec. IV. In that section it is shown how the use of the Ward identities in tensor models permits us to write Gaussian correlators as correlators involving the cut operator as in Eq. (4.5). This is crucial for the emergence of a notion of locality in tensor models: hitting as a derivative, the cut operator acts on Schur invariants deleting a corner box in each of the invariant's labels. Those distinguished corners can be mapped to the grid, which, on the gravity side, is discretized space. The most important result of Sec. IV, and perhaps of the paper, is Eq. (4.14). Finally, in Sec. IV C, I propose a tensor quantity which seems reasonable to relate to the ADM mass of spacetimes.

\section{TENSOR MODELS}

In this section, I review known facts about tensor models and set the notation. I also define the invariants which will play a role in the partition function and will be relevant in this paper. More information can be found in the Appendixes and in the references provided.

\section{A. Invariants}

The basic object of color tensor models is the tensor $T$ of order $d$ and size $N$. The tensor $T$ is a box of $N^{d}$ complex numbers whose components transform under the gauge group $U(N)^{\times d}$ as

$$
T_{j_{1} j_{2} \ldots j_{d}}=\sum_{i_{1}, \ldots, i_{d}} U_{1}(N)_{j_{1}}^{i_{1}} \cdots U_{d}(N)_{j_{d}}^{i_{d}} T_{i_{1} i_{2} \ldots i_{d}}
$$

where, with $U_{i}(N)$, I am emphasizing that each component transforms under a different copy of $U(N)$. The complex conjugate is a contravariant tensor that transforms as

$$
\bar{T}^{j_{1} j_{2} \ldots j_{d}}=\sum_{i_{1}, \ldots, i_{d}} \bar{U}_{1}(N)_{i_{1}}^{j_{1}} \cdots \bar{U}_{d}(N)_{i_{d}}^{j_{d}} \bar{T}^{i_{1} i_{2} \ldots i_{d}}
$$

Invariants under $U(N)^{\times d}$ are made of $n$ copies of $T$ and $n$ copies of $\bar{T}$ as we contract all the indices of the tensors by pairs $(T, \bar{T})$ respecting the index position. So, first indices contract only with first indices, and so on. Any possible invariant can be obtained as a linear combination of the elements of the set

$$
\left\{\mathcal{O}_{\alpha_{1} \ldots \alpha_{d}}=\prod_{p=1}^{n} T_{i_{1}^{p} i_{2}^{p} \ldots i_{d}^{p}} \bar{T}^{i_{1}^{\alpha_{1}(p)}} i_{2}^{\alpha_{2}(p)} \ldots i_{d}^{\alpha_{d}(p)} \mid\left(\alpha_{1} \ldots \alpha_{d}\right) \in S_{n}^{d}\right\}
$$

where subscripts and superscripts have been assigned to indices in order to specify the location of the component and the slot that each tensor occupies in the string of $n$ copies. As the notation in tensor theory quickly proliferates, for practical reasons I will reduce it as much as possible by omitting indices whenever they are not strictly necessary. The set (2.3) has often been called the permutation basis in the literature; see [11].

\section{B. Notation}

In addition to the usual notation for tensor models, I will adopt a vector notation for $d$-tuples

$$
\begin{array}{ll}
\vec{\alpha}=\left(\alpha_{1}, \ldots, \alpha_{d}\right), & \alpha_{i} \in S_{n}, \\
\vec{\mu}=\left(\mu_{1}, \ldots, \mu_{d}\right), & \mu_{i} \vdash n,
\end{array}
$$

where $\alpha_{i}$ are permutations from the symmetric group $S_{n}$. Thus, $\vec{\alpha}$ is an element of the group $S_{n}^{d}$. The symbol " $\vdash$ " represents partition, so $\mu \vdash n$ indicates that $\mu$ is a partition of $n$. Remember that partitions of $n$ label irreducible representations of $S_{n}$. This way, $\vec{\mu}$ is a collection of $d$ partitions of $n$, which labels an irreducible representation of $S_{n}^{d}$. The number of cycles of a given permutation will be written as $C(\alpha)$. If it is applied to a collection of permutations, then

$$
C(\vec{\alpha})=\sum_{i} C\left(\alpha_{i}\right)
$$

The product of two elements of $S_{n}^{d}$ and a diagonal product of an element of $S_{n}$ with an element of $S_{n}^{d}$ will be written as

$\vec{\alpha} \cdot \vec{\beta}=\left(\alpha_{1} \beta_{1}, \ldots, \alpha_{d} \beta_{d}\right) \quad$ and $\quad \vec{\alpha} \cdot \sigma=\left(\alpha_{1} \sigma, \ldots, \alpha_{d} \sigma\right)$,

respectively. This vector notation applies to every mathematical object found in this paper. For instance, central objects in this work are the characters of the symmetric group, denoted as $\chi_{\mu}(\alpha)$ when it is the character of the irreducible representation $\Gamma_{\mu}$ of the permutation $\alpha$. Then, following the logic of the vector notation, we will write for a product of characters

$$
\chi_{\vec{\mu}}(\vec{\alpha})=\chi_{\mu_{1}}\left(\alpha_{1}\right) \cdots \chi_{\mu_{d}}\left(\alpha_{d}\right)
$$


Quantities like the Littlewood-Richardson coefficients which, in terms of characters of the symmetric group, can be expressed as

$$
\begin{gathered}
C_{\mu \nu}^{\lambda}=\frac{1}{n ! m !} \sum_{\sigma \in S_{n}} \sum_{\tau \in S_{m}} \chi_{\mu}(\sigma) \chi_{\nu}(\tau) \chi_{\lambda}(\sigma \circ \tau), \\
\mu \vdash m, \quad \nu \vdash n, \quad \lambda \vdash n+m,
\end{gathered}
$$

where the product $\sigma \circ \tau$ indicates that the permutations are disjoint, are also subjected to vector notation. So,

$$
\begin{aligned}
C_{\vec{\mu} \vec{\nu}}^{\vec{\lambda}} & =C_{\mu_{1} \nu_{1}}^{\lambda_{1}} \cdots C_{\mu_{d} \nu_{d}}^{\lambda_{d}}, \quad \mu_{i} \vdash m, \\
& \nu_{i} \vdash n, \quad \lambda_{i} \vdash n+m .
\end{aligned}
$$

For dimensions of the representations of the symmetric group and the unitary group I will write, respectively,

$$
\begin{aligned}
d_{\vec{\mu}} & =d_{\mu_{1}} \cdots d_{\mu_{1}} \quad \text { and } \\
\operatorname{Dim}_{\vec{\mu}}(N) & =\operatorname{Dim}_{\mu_{1}}(N) \cdots \operatorname{Dim}_{\mu_{d}}(N) .
\end{aligned}
$$

It is well known that the dimensions of the representations of the symmetric group as well as the representations of the unitary group can be computed combinatorially. They read

$$
d_{\mu}=\frac{n !}{\operatorname{Hooks}_{\mu}}
$$

and

$$
\operatorname{Dim}_{\lambda}(N)=\frac{1}{n !} f_{\lambda}(N) d_{\lambda},
$$

respectively, where

$$
f_{\lambda}(N)=\prod_{(i, j) \in \lambda}(N+j-i) .
$$

We will also write

$$
f_{\vec{\mu}}(N)=f_{\mu_{1}}(N) \cdots f_{\mu_{d}}(N) .
$$

Quantities like the Kronecker coefficients will be written in vector shorthand notation as well. So, I will write

$$
g_{\vec{\mu}}=g_{\mu_{1} \ldots \mu_{d}}=\frac{1}{n !} \sum_{\sigma \in S_{n}} \chi_{\mu_{1}}(\sigma) \cdots \chi_{\mu_{d}}(\sigma) .
$$

Trace and Schur operators will be denoted as

$$
\mathcal{O}_{\vec{\alpha}}=\mathcal{O}_{\alpha_{1} \ldots \alpha_{d}}, \quad \mathcal{O}_{\vec{\mu} ; i j}=\mathcal{O}_{\mu_{1} \ldots \mu_{d} ; i j}
$$

In this paper, I will generally use prime greek letters to represent objects related to $n-1$ elements. I find it especially convenient when dealing with the cut operation.
There, I will call $\alpha^{\prime}$ the permutation of $n-1$ elements that results from $\alpha$ after deleting the letter "n." The same logic will be used for Young diagrams, where the notation $\mu^{\prime} \nearrow \mu$ means that the diagram $\mu^{\prime} \vdash n-1$ is obtained from $\mu$ after deleting one corner box. This notation will be extended vectorially; thus,

$$
\overrightarrow{\mu^{\prime}} \nearrow \vec{\mu}
$$

will refer to a $d$-tuple of diagrams $\overrightarrow{\mu^{\prime}}$ that is obtained from the $d$-tuple $\vec{\mu}$ after deleting one corner box in each $\mu_{i}$.

\section{Restricted Schur basis and Schur invariants}

Despite the name, the elements of the set (2.3), although they span the space of invariants, do not form a basis. They overexpress the space of invariants. This is not merely because of the obvious equivalence

$$
\mathcal{O}_{\tau \cdot \vec{\alpha} \cdot \sigma}=\mathcal{O}_{\vec{\alpha}}
$$

a redundancy that may be removed by considering only double coset representatives, but also because the elements of Eq. (2.3) are not linearly independent for $n>N$. Using arguments of representation theory, the exact number of invariants for given $N$ and $n$ was found ${ }^{4}[12,13,15,20]$ and the natural basis adapted to the counting, the restricted Schur basis, was constructed ${ }^{5}$; see Appendix A 2 for details. It is the set

$\left\{\mathcal{O}_{\vec{\mu} ; i j} \mid \quad \mu_{i} \vdash n, \quad l\left(\mu_{i}\right) \leq N, \quad i, j=1, \ldots, g_{\vec{\mu}}\right\}$.

The condition $l\left(\mu_{i}\right) \leq N$ forces each Young diagram $\mu_{i}$ to have a maximum of $N$ rows. The elements of the basis can be written as

$$
\mathcal{O}_{\vec{\mu} ; i j}=\sum_{\vec{\alpha} \in S_{n}^{d}} F_{\vec{\mu} ; i j}(\vec{\alpha}) \mathcal{O}_{\vec{\alpha}}
$$

for suitable complex double coset invariant functions $F_{\vec{\mu} ; i j}(\vec{\alpha})$ which fulfill the convolution algebra

$$
F_{\vec{\mu} ; i j} * F_{\vec{\nu} ; k l}(\vec{\alpha})=\delta_{\vec{\mu} \vec{\nu}} \delta_{j k} F_{\vec{\mu} ; i l}(\vec{\alpha}) .
$$

The functions $F_{\vec{\mu} ; i j}(\vec{\alpha})$ are projectors with the labels $\vec{\mu}$ and intertwiners with the labels $i j$. For reasons that will become clear later, in this paper we will be interested in a subset of

\footnotetext{
${ }^{4}$ See also [30,31] for the counting of invariants in tensor theories with an orthogonal gauge group.

${ }^{5}$ Analogous bases of operators have been constructed in matrix models, first [32] for a single matrix model, and later [33,34] for multimatrix models. See also $[35,36]$ for other multimatrix model bases.
} 
invariants which we will call Schur invariants, ${ }^{6}$ and which are defined as

$$
\mathcal{O}_{\vec{\mu}} \equiv \sum_{i=1}^{g_{\vec{\mu}}} \mathcal{O}_{\vec{\mu} ; i i}
$$

Schur invariants are built out of projectors [see Eq. (A10)] and can be constructed explicitly. They are

$$
\mathcal{O}_{\vec{\mu}}=\frac{1}{n !^{d-1}} \sum_{\vec{\alpha} \in S_{n}^{d}} d_{\vec{\mu}} \chi_{\vec{\mu}}(\vec{\alpha}) \mathcal{O}_{\vec{\alpha}}
$$

Note that the normalization of the invariants $\mathcal{O}_{\vec{\mu}}$ differs from the usual choices, as in [21]. The reason for this is that with the normalization of Eq. (2.23), the invariants correspond to true projectors in the space of coset invariant functions. See Appendix A 1 for details.

\section{Action, partition function, and correlators}

The partition function of the theory is

$$
Z[\lambda]=\int d T d \bar{T} \exp \left(-\frac{N^{d-1}}{2} S[T, \bar{T}]\right) .
$$

The letter $\lambda$ encodes the couplings of all the interacting terms. The factor $N^{d-1}$ in front of the action makes the model asymptotically free as $N \rightarrow \infty$ [38].

The most general action, which includes all the invariant operators of the theory, is

$$
S[T, \bar{T}]=T \cdot \bar{T}+\frac{1}{N^{d-1}} \sum_{\vec{\mu}, i j} \lambda_{\vec{\mu}, i j} \mathcal{O}_{\vec{\mu}, i j}
$$

In this paper, we will be considering the sector of Schur invariants, so the action we will use is

$$
S[T, \bar{T}]=T \cdot \bar{T}+\frac{1}{N^{d-1}} \sum_{\vec{\mu}} \lambda_{\vec{\mu}} \mathcal{O}_{\vec{\mu}} .
$$

With Eq. (2.24), the two-point function for the free theory of single tensors reads

$$
\left\langle T_{i_{1} \ldots i_{d}} \bar{T}^{j_{1} \ldots j_{d}}\right\rangle_{0}=\frac{1}{N^{d-1}} \delta_{i_{1}}^{j_{1}} \cdots \delta_{i_{d}}^{j_{d}}
$$

where the subscript " 0 " indicates a Gaussian average, with no subscript indicating that the average involves the full action (2.26). The correlator of the trace operators made of $2 n$ tensors is

\footnotetext{
${ }^{6}$ Also called permutation centralizer algebras (see [37]) and Kronecker tensorial character (see [21]) in the literature.
}

$$
\left\langle\mathcal{O}_{\vec{\alpha}}\right\rangle_{0}=\frac{1}{N^{n(d-1)}} \sum_{\sigma \in S_{n}} N^{C(\vec{\alpha} \cdot \sigma)},
$$

with the definition of the number of cycles given in Eq. (2.5). Applying the formula

$$
N^{C(\sigma)}=\sum_{\mu \vdash n} \chi_{\mu}(\sigma) \operatorname{Dim}_{\mu}(N)
$$

and the orthogonality of characters, it is straightforward to see that the Gaussian average of Schur invariants $\mathcal{O}_{\vec{\mu}}$ is

$$
\left\langle\mathcal{O}_{\vec{\mu}}\right\rangle_{0}=\frac{n !}{N^{n(d-1)}} \operatorname{Dim}_{\vec{\mu}}(N) g_{\vec{\mu}}=\frac{1}{n !^{d-1}} \frac{1}{N^{n(d-1)}} f_{\vec{\mu}}(N) d_{\vec{\mu}} g_{\vec{\mu}},
$$

where $f_{\vec{\mu}}(N)$ has been given in Eq. (2.14) with Eq. (2.13) and $g_{\vec{\mu}}$ are the Kronecker coefficients defined in Eq. (2.15).

\section{CONNECTION WITH GRAVITY AT THE SADDLE POINT}

Via triangulations, a close relation has been established between tensor models and gravity. Every invariant built on $2 n(d+1)$ tensors can be associated with a triangulation with $2 n d$-simplices where the pattern of contraction of the indices encodes the details of the triangulation. The Wick contractions of observables built on a $2 n$ tensor of order $d$ can be interpreted as invariants of order $d+1$, which may be associated with triangulations made of $2 n d$-simplices. Remarkably, it has been proven [24] that, provided the appropriate identifications, the statistics of tensor models match those of triangulations driven by Regge calculus, what establishes a solid connection between tensor models and gravity at the level of partition functions.

It is not easy to find the tensor sector that corresponds to the continuum limit for gravity, that is, classical gravity. It will happen at large $N$, and there is a common belief that it should be at a fixed point of a certain renormalization flow; see [39] and the references therein. This way, classical gravity would be sitting at a universality class where any detail of a specific triangulation would be irrelevant, as it should be. Then, we recover the necessary symmetry under diffeomorphisms of classical gravity. ${ }^{7}$ The big question is whether tensor invariants could also, in some large limit of $n$ and $N$, encode a background, a classical solution of gravity. The main goal of this paper is to propose a collection of invariants (Schur invariants) which can be associated with backgrounds, as well as to establish a precise relation between them.

\footnotetext{
${ }^{7}$ Different triangulations reduce to different coordinate systems at the continuum limit of Regge calculus.
} 


\section{A. Schur invariants and backgrounds}

I claim that Schur invariants correspond to backgrounds. This claim is supported by the factorization of the threepoint function at large $n, N$ in the presence of a Schur invariant. Let us define

$$
\left\langle\left\langle\mathcal{O}_{\vec{\alpha}}\right\rangle\right\rangle_{\vec{\mu}} \equiv \frac{\left\langle\mathcal{O}_{\vec{\alpha}} \mathcal{O}_{\vec{\mu}}\right\rangle_{0}}{\left\langle\mathcal{O}_{\vec{\mu}}\right\rangle_{0}}
$$

where $\vec{\mu}$ labels a Schur invariant with $\mu_{i} \vdash n$ and $\mathcal{O}_{\vec{\alpha}}$ is an invariant made of a few (order 1) tensors. Thus, $\mathcal{O}_{\vec{\alpha}}$ will be interpreted as an excitation of the background $\mathcal{O}_{\vec{\mu}}$. For the classical behavior of the background, we need to prove that, for large $n, N$,

$$
\left\langle\left\langle\mathcal{O}_{\vec{\alpha}} \mathcal{O}_{\vec{\beta}}\right\rangle\right\rangle_{\vec{\mu}} \approx\left\langle\left\langle\mathcal{O}_{\vec{\alpha}}\right\rangle\right\rangle_{\vec{\mu}}\left\langle\left\langle\mathcal{O}_{\vec{\beta}}\right\rangle\right\rangle_{\vec{\mu}}
$$

Condition (3.2) assures the independence of the states $\mathcal{O}_{\vec{\alpha}}$ and $\mathcal{O}_{\vec{\beta}}$ when they happen in the large "environment" $\mathcal{O}_{\vec{\mu}}$. Consequently, the density matrix of any "multiparticle" state turns diagonal. For this reason, I will assume that if the condition (3.2) holds for any $\mathcal{O}_{\vec{\alpha}}$ and $\mathcal{O}_{\vec{\beta}}$, then $\mathcal{O}_{\vec{\mu}}$ is a background and $\mathcal{O}_{\vec{\alpha}}$ and $\mathcal{O}_{\vec{\beta}}$ should be thought of as excitations of $\mathcal{O}_{\vec{\mu}}$. For simplicity I am also going to consider $\mathcal{O}_{\vec{\alpha}}$ and $\mathcal{O}_{\vec{\beta}}$ Schur invariants with numbers of tensor copies $n_{1}$ and $n_{2}$, respectively, where $n_{1}, n_{2} \ll n$. So, one should think of $\mathcal{O}_{\vec{\alpha}}$ and $\mathcal{O}_{\vec{\beta}}$ as (Schur) excitations of $\mathcal{O}_{\vec{\mu}}$.

With the definition of Schur invariants (2.23) and their averages (2.30) and applying (B1) for the product of two Schur invariants, we have

$$
\begin{aligned}
\left\langle\mathcal{O}_{\vec{\alpha}} \mathcal{O}_{\vec{\beta}} \mathcal{O}_{\vec{\mu}}\right\rangle_{0}= & \frac{n ! n_{1} ! n_{2} !}{\left(n+n_{1}+n_{2}\right) !^{d}} \frac{d_{\vec{\alpha}} d_{\vec{\beta}} d_{\vec{\mu}}}{N^{\left(n+n_{1}+n_{2}\right)(d-1)}} \\
& \times \sum_{\lambda_{i} \vdash n+n_{1}+n_{2}} f_{\vec{\lambda}} g_{\vec{\lambda}} C_{\vec{\mu} \vec{\alpha} \vec{\beta}}^{\vec{\lambda}}
\end{aligned}
$$

and

$$
\begin{aligned}
\frac{\left\langle\mathcal{O}_{\vec{\alpha}} \mathcal{O}_{\vec{\mu}}\right\rangle_{0}\left\langle\mathcal{O}_{\vec{\beta}} \mathcal{O}_{\vec{\mu}}\right\rangle_{0}}{\left\langle\mathcal{O}_{\vec{\mu}}\right\rangle_{0}}= & \frac{n !^{d} n ! n_{1} ! n_{2} !}{\left(n+n_{1}\right) !^{d}\left(n+n_{2}\right) !^{d}} \frac{d_{\vec{\alpha}} d_{\vec{\beta}} d_{\vec{\mu}}}{N^{\left(n+n_{1}+n_{2}\right)(d-1)}} \\
& \times \sum_{\substack{\lambda^{\prime} \vdash n+n_{1} \\
\lambda_{i}^{\prime \prime} \vdash n+n_{2}}} \frac{f_{\vec{\lambda}^{\prime}} f_{\vec{\lambda}^{\prime \prime}}}{f_{\vec{\mu}}} \frac{g_{\overrightarrow{\lambda^{\prime}}} g_{\vec{\lambda}^{\prime \prime}}}{g_{\vec{\mu}}} C_{\vec{\mu} \vec{\alpha}}^{\vec{\lambda}^{\prime}} C_{\vec{\mu} \vec{\mu}}^{\vec{\lambda}^{\prime \prime}}, \quad \text { (3.4) }
\end{aligned}
$$

where all the quantities involved have been defined in Sec. II B.

As commented above, to claim that $\mathcal{O}_{\vec{\mu}}$ is a background we must prove that Eqs. (3.3) and (3.4) are equal at $n, N \rightarrow \infty$. A rigorous proof of this statement in full generality is challenging because of the difficulties one encounters when dealing with the Kronecker coefficients.
However, I will offer a check, valid for some configurations $\vec{\mu}$, that clearly supports the statement.

The key property which lies under the factorization of Eq. (3.3) into Eq. (3.4) is the factorization of normalized characters of the symmetric group at large $n .^{8}$ That is,

$$
\bar{\chi}_{\mu}\left(\sigma_{1} \circ \sigma_{2}\right)=\bar{\chi}_{\mu}\left(\sigma_{1}\right) \bar{\chi}_{\mu}\left(\sigma_{2}\right)+o(1 / n),
$$

where the normalized character is defined as

$$
\bar{\chi}_{\lambda}(\sigma) \equiv \frac{\chi_{\lambda}(\sigma)}{d_{\lambda}} .
$$

The property (3.5) come from the explicit form of the characters for large $n$ found by Biane [40],

$\bar{\chi}_{\lambda}(\sigma)=C_{\sigma}(w) n^{-|\sigma| / 2}+O\left(n^{-|\sigma| / 2-1}\right), \quad \lambda \vdash n$,

where $|\sigma|$ is the minimal number of transpositions necessary to generate $\sigma$ and $w$ is the limit shape that the partition $\lambda$ approaches. The crucial fact in formula (3.7) is that $C_{\sigma \circ \tau}(w)=C_{\sigma}(w) C_{\tau}(w)$; that is, the function factorizes whenever $\sigma$ and $\tau$ are disjoint permutations. It is also of special note that $C_{\sigma}(w)$ depends only on the limit shape $w$ and not on the particular partition $\lambda$, a fact that I will use later.

Because of (3.5), the Littlewood-Richardson numbers adopt the useful form

$$
C_{\mu \nu}^{\lambda}=\frac{1}{n ! n_{1} !} \sum_{\substack{\sigma \in S_{n} \\ \sigma_{1} \in S_{n_{1}}}} \chi_{\lambda}\left(\sigma \circ \sigma_{1}\right) \chi_{\mu}(\sigma) \chi_{\nu}\left(\sigma_{1}\right) \sim \frac{1}{d_{\lambda}} d_{\lambda / \mu} d_{\lambda / \nu}
$$

and

$$
\begin{aligned}
C_{\mu \nu \rho}^{\lambda} & =\frac{1}{n ! n_{1} ! n_{2} !} \sum_{\substack{\sigma \in S_{n} \\
\sigma_{1} \in S_{n_{1}} \\
\sigma_{2} \in S_{n_{2}}}} \chi_{\lambda}\left(\sigma \circ \sigma_{1} \circ \sigma_{2}\right) \chi_{\mu}(\sigma) \chi_{\nu}\left(\sigma_{1}\right) \chi_{\rho}\left(\sigma_{2}\right) \\
& \sim \frac{1}{d_{\lambda}^{2}} d_{\lambda / \mu} d_{\lambda / \nu} d_{\lambda / \rho},
\end{aligned}
$$

where

$d_{\lambda / \mu}=\frac{1}{n !} \sum_{\sigma \in S_{n}} \chi_{\lambda}(\sigma) \chi_{\mu}(\sigma), \quad \lambda \vdash n+n_{1}, \mu \vdash n$.

The number $d_{\lambda / \mu}$ is called the relative dimension. It is the number of times that the representation $\Gamma_{\mu}$ is subduced from $\Gamma_{\lambda}$ when the group is restricted from $S_{n+n_{1}}$ to $S_{n}$. The number $d_{\lambda / \mu}$ also counts the paths that join the partition $\lambda$ and the partition $\mu$ in the Young graph or, equivalently, the number of partially labeled Young

\footnotetext{
${ }^{8}$ See [40].
} 
diagrams between $\lambda$ and $\mu$. Of course, this number will be 0 if $\mu$ is not subduced by $\lambda$. Usually, the computation of Littlewood-Richardson (LR) numbers, although it can be done combinatorially, is much more complicated than in Eq. (3.8) and involves a precise relation between partitions $\mu$ and $\nu$ besides their individual relation with $\lambda$. However, as seen in (3.8), this contribution is subleading at large $n$.

Let us see how Eq. (3.3) approaches Eq. (3.4) at large $n$, $N$. The first thing to notice is that the prefactors in front of their respective sums are equal at large $n$, so I will not worry about them in the following. We now have

$$
\begin{aligned}
& \sum_{\lambda_{i} \vdash n+n_{1}+n_{2}} C_{\vec{\mu} \vec{\alpha} \vec{\beta}}^{\vec{\lambda}} f_{\vec{\lambda}} g_{\vec{\lambda}} \approx \sum_{\lambda_{i} \vdash n+n_{1}+n_{2}} d_{\vec{\lambda} / \vec{\mu}} \frac{d_{\vec{\lambda} / \vec{\alpha}}}{d_{\vec{\lambda}}} \frac{d_{\vec{\lambda} / \vec{\beta}}}{d_{\vec{\lambda}}} f_{\vec{\lambda}} g_{\vec{\lambda}}
\end{aligned}
$$

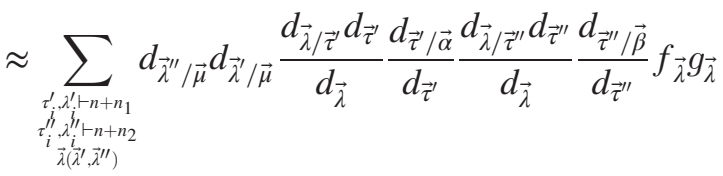

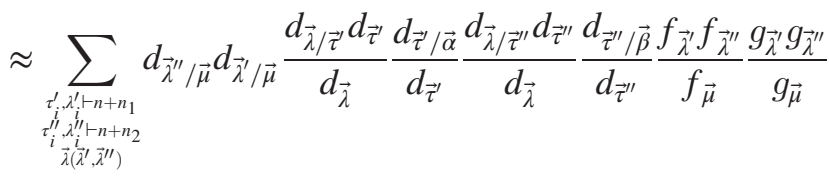

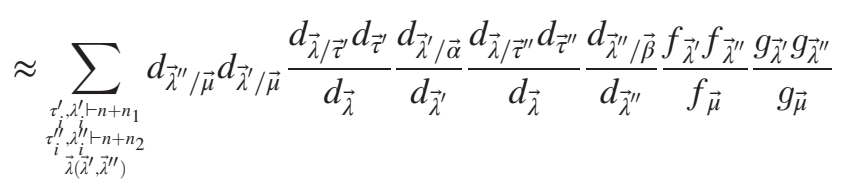

$$
\begin{aligned}
& =\sum_{\substack{\lambda^{\prime} \vdash n+n_{1} \\
\lambda_{i}^{\prime \prime} \vdash n+n_{2}}} d_{\vec{\lambda}^{\prime \prime} / \vec{\mu}} d_{\vec{\lambda}^{\prime} / \vec{\mu}} \frac{d_{\vec{\lambda}^{\prime}} / \vec{\alpha}}{d_{\vec{\lambda}^{\prime}}} \frac{d_{\vec{\lambda}^{\prime \prime} / \vec{\beta}}}{d_{\vec{\lambda}^{\prime \prime}}} \frac{f_{\vec{\lambda}^{\prime}} f_{\vec{\lambda}^{\prime \prime}}}{f_{\vec{\mu}}} \frac{g_{\vec{\lambda}^{\prime}} g_{\vec{\lambda}^{\prime \prime}}}{g_{\vec{\mu}}} \\
& \approx \sum_{\substack{\lambda^{\prime} \vdash n+n_{1} \\
\lambda_{i}^{\prime \prime} \vdash n+n_{2}}} C_{\vec{\mu} \vec{\alpha}} C_{\vec{\mu}}^{\vec{\lambda}^{\prime} \vec{\beta}} \frac{f_{\vec{\lambda}^{\prime}} f_{\vec{\lambda}^{\prime \prime}}}{f_{\vec{\mu}}} \frac{g_{\vec{\lambda}^{\prime}} g_{\vec{\lambda}^{\prime \prime}}}{g_{\vec{\mu}}},
\end{aligned}
$$

and then

$$
\left\langle\mathcal{O}_{\vec{\alpha}} \mathcal{O}_{\vec{\beta}} \mathcal{O}_{\vec{\mu}}\right\rangle_{0} \approx \frac{\left\langle\mathcal{O}_{\vec{\alpha}} \mathcal{O}_{\vec{\mu}}\right\rangle_{0}\left\langle\mathcal{O}_{\vec{\beta}} \mathcal{O}_{\vec{\mu}}\right\rangle_{0}}{\left\langle\mathcal{O}_{\vec{\mu}}\right\rangle_{0}},
$$

where the equality is reached at the limit $n \rightarrow \infty$.

Let us explain the approaches taken in Eqs. (3.11)-(3.16).

In Eq. (3.11), I have used Eq. (3.9) to convert, at large $n$, the LR numbers into the dimension and relative dimensions of the irreducible representations associated with the Young graph, the branching graph of the symmetric groups.
In Eq. (3.12), I have used the chain property ${ }^{9}$ of the Young graph

$$
d_{\lambda / \alpha}=\sum_{\tau \vdash n^{\prime \prime}} d_{\lambda / \tau} d_{\tau / \alpha}, \quad \lambda \vdash n \quad \alpha \vdash n^{\prime},
$$

which is valid for all $n \geq n^{\prime \prime} \geq n^{\prime}$.

I have also approached the relative dimensions for large partitions. Given the Young diagrams $\lambda \vdash n+n_{1}+n_{2}$ and $\mu \vdash n$, the relative dimension for large $n$ is well approximated by $\left(n_{1}+n_{2}\right)$ !, and analogously for $\lambda^{\prime} \vdash n+n_{1}$ and $\lambda^{\prime \prime} \vdash n+n_{2}$. So,

$$
d_{\lambda / \mu} \approx \frac{\left(n_{1}+n_{2}\right) !}{n_{1} ! n_{2} !} d_{\lambda^{\prime} / \mu} d_{\lambda^{\prime \prime} / \mu}
$$

In order to turn a sum over $\lambda$ into a sum over $\lambda^{\prime}$ and $\lambda^{\prime \prime}$, we connect them as follows. The Young diagram $\lambda^{\prime}$ is obtained from $\mu$ by adding $n_{1}$ boxes. We mark the corners of $\mu$ where these boxes are added. We do the same for $\lambda^{\prime \prime}$, which is obtained from $\mu$ with the addition of $n_{2}$ boxes. Now we construct $\lambda\left(\lambda^{\prime}, \lambda^{\prime \prime}\right)$ by adding $n_{1}+n_{2}$ boxes to $\mu$ in the indicated corners. Be aware that to each $\lambda$ corresponds $\left(\begin{array}{c}n_{1}+n_{2} \\ n_{1}\end{array}\right)$ different choices of $\lambda^{\prime}$ and $\lambda^{\prime \prime}$. So, the sum over $\lambda^{\prime}$ and $\lambda^{\prime \prime}$ overcounts the sum over $\lambda$ by the factor $\left(\begin{array}{c}n_{1}+n_{2} \\ n_{1}\end{array}\right)$. This combinatoric number should divide the sum (3.12), but it exactly cancels the prefactor in Eq. (3.19) and, consequently, no extra factors appear in Eq. (3.12).

In Eq. (3.13), I used Eq. (2.13) and the correspondence $\lambda\left(\lambda^{\prime}, \lambda^{\prime \prime}\right)$ described in the previous paragraph to write

$$
f_{\lambda}=\frac{f_{\lambda^{\prime}} f_{\lambda^{\prime \prime}}}{f_{\mu}}
$$

For the Kronecker coefficients, the approach is taken under the assumption that the states labeled by $\vec{\mu}$ are typical states, so the limit shapes are close to the Plancherel curve, where the highest dimension for representations is reached and Kronecker coefficients are also maximal [42]. The word "typical" refers to the Plancherel measure

$$
P[\lambda]=\frac{d_{\lambda}^{2}}{n !}, \quad \frac{1}{n !} \sum_{\lambda \vdash n} d_{\lambda}^{2}=1,
$$

which is naturally associated with the branching graph of the symmetric group. Related to this measure, the probability that we pick the Plancherel curve as the limit shape as $n \rightarrow \infty$ is 1 .

The value of the Kronecker coefficients for order 3 tensors when the limit shape is close to the Plancherel curve is

\footnotetext{
${ }^{9}$ See [41].
} 


$$
g_{\mu_{1} \mu_{2} \mu_{3}} \approx \sqrt{n !} \exp \left(-a_{p} n\right), \quad \mu_{i} \vdash n, \quad a_{p} \geq 0,
$$

where the equality is reached when all the partitions approach the Plancherel limit shape. This justifies

$$
g_{\vec{\lambda}} \approx \frac{g_{\vec{\lambda}^{\prime}} g_{\vec{\lambda}^{\prime \prime}}}{g_{\vec{\mu}}},
$$

which was used in Eq. (3.13). As commented above, the approach of Kronecker coefficients (3.23) is modest since it is restricted to limit shapes close to the Plancherel curve. I claim that the factorization of the three-point function (3.17) occurs for any limit shape, so a definite proof has to involve a generalization of Eq. (3.23) valid for generic limit shapes.

In Eq. (3.14), the only changes I introduce are

$$
\frac{d_{\vec{\tau}^{\prime}} / \vec{\alpha}}{d_{\vec{\tau}^{\prime}}} \rightarrow \frac{d_{\vec{\lambda}^{\prime} / \vec{\alpha}}}{d_{\vec{\lambda}^{\prime}}} \quad \text { and } \quad \frac{d_{\vec{\tau}^{\prime \prime} / \vec{\beta}}}{d_{\vec{\tau}^{\prime \prime}}} \rightarrow \frac{d_{\vec{\lambda}^{\prime \prime} / \vec{\beta}}}{d_{\vec{\lambda}^{\prime \prime}}} .
$$

Be aware that, from Eq. (3.10), we see that

$$
\begin{aligned}
\frac{d_{\tau^{\prime} / \alpha}}{d_{\tau^{\prime}}} & =\frac{1}{n_{1} !} \sum_{\sigma \in S_{n_{1}}} \bar{\chi}_{\tau^{\prime}}(\sigma) \chi_{\alpha}(\sigma) \\
& \approx \frac{1}{n_{1} !} \sum_{\sigma \in S_{n_{1}}} C_{\sigma}(w) n^{-|\sigma| / 2} \chi_{\alpha}(\sigma) \\
& \approx \frac{1}{n_{1} !} \sum_{\sigma \in S_{n_{1}}} \bar{\chi}_{\lambda^{\prime}}(\sigma) \chi_{\alpha}(\sigma)=\frac{d_{\lambda^{\prime} / \alpha}}{d_{\lambda^{\prime}}} .
\end{aligned}
$$

The key point in Eq. (3.25) is that both $\tau^{\prime}$ and $\lambda^{\prime}$ differ in $n_{1}$ boxes from $\mu$, and they have the same limit shape $w$, which is assured as long as $n_{1} \ll n$.

Finally, in Eq. (3.15), I used the stochastic property of the relative dimensions [41], by means of which

$$
\sum_{\tau^{\prime}} \frac{d_{\lambda / \tau^{\prime}} d_{\tau^{\prime}}}{d_{\lambda}}=1 \quad \forall \lambda
$$

Note that the factorization of the three-point function (3.17) is a very nontrivial statement. The factorization properties of characters at large $n$ play a crucial role in this approach. The product of Schur invariants involves LR numbers, whose approximation (3.8) at large $n$ is at the core of the proof. As I said, Schur invariants are perhaps not the only ones that are entitled to partner backgrounds, but I cannot easily think of other invariants that fulfill Eq. (3.17) and that can be proven to do so.

\section{B. Path integral ansatz}

Although it is not clear-and certainly not proven in this article-that Schur invariants are the only large states which can be traded as backgrounds, we find it convenient to restrict ourselves to this subspace of invariants. The reason is twofold: on the one hand, I have just proven in Eq. (3.17) that they behave appropriately at large $n$, and, on the other hand, that they are easier to operate with since we can construct them explicitly.

In the following I am going to be loyal to two ideas:

(1) I will take seriously the idea that tensor models encode quantum gravity. This is a reasonable assumption given the success of tensor models in describing discretized quantum gravity via triangulations.

(2) I will associate a background to a Schur operator $\mathcal{O}_{\vec{\mu}}$. This is analogous to the usual association trace invariant $\leftrightarrow$ PL manifold. However, as opposed to the triangulation scheme, it is not obvious how to make the association Schur operator $\leftrightarrow$ PL manifold a priori.

As said above, the interacting terms I will be considering in the action are Schur invariants. Thus, the action will be

$$
S[T, \bar{T}]=T \cdot \bar{T}+\frac{1}{N^{d-1}} \sum_{\vec{\mu}} \lambda_{\vec{\mu}} \mathcal{O}_{\vec{\mu}} .
$$

Therefore, the partition function of the tensor model I am considering is Eq. (2.24) with Eq. (3.27). In my proposal, the partition function of the tensor model should be equated to the partition function of gravity, so one would like to schematically write

$$
Z[\lambda]=Z_{g}[\kappa]=\int d g \exp \left(-S_{\kappa}[g]\right),
$$

where $\kappa$ is a label for the higher derivative terms of the gravity action. In order to make sense of Eq. (3.28), let us examine the region near a solution of the gravity equations, that is, near a background. In view of the correspondence between Schur invariants and backgrounds, and with a slight abuse of notation, let us refer to the background as $\vec{\mu}$ when it is associated with the tensor invariant $\mathcal{O}_{\vec{\mu}}$. Accordingly, I will write $S[\vec{\mu}]$ for the on-shell gravity action on the background $\vec{\mu}$. Near this background, the gravity path integral can be well approximated as

$$
Z_{g}[\kappa]=e^{-S_{\kappa}[\vec{\mu}]} .
$$

On the tensor side of the equality (3.28), we can write

$$
Z[\lambda]=\left\langle\exp \left(\sum_{\vec{\mu}} \lambda_{\vec{\mu}} \mathcal{O}_{\vec{\mu}}\right)\right\rangle_{0},
$$

where the subscript 0 reminds us that the average is Gaussian. Note that in Eq. (3.30) there is no Schur invariant chosen: the sum is over all of them. In order 
to match the saddle point approach of Eq. (3.29) to its tensor counterpart, we have to impose a certain "projection" of $Z[\lambda]$ onto $\mathcal{O}_{\vec{\mu}}$. I will write

$$
P_{\vec{\mu}}(Z[\lambda])=e^{-S_{\kappa}[\vec{\mu}]} .
$$

For the purpose of finding such a projection, let us remember that since $N$ must be large to make contact with classical gravity and because tensor models are asymptotically free [38], the couplings $\lambda_{\vec{\mu}}(N)$ must be small and a Taylor expansion on them is expected to be accurate with a few terms. So, let us Taylor expand Eq. (3.30). We will have

$$
Z[\lambda]=\sum_{n \geq 0} \frac{1}{n !}\left\langle\left(\sum_{\vec{\nu}} \lambda_{\vec{\nu}} \mathcal{O}_{\vec{\nu}}\right)^{n}\right\rangle_{0} .
$$

The first terms of this expansion read

$$
Z[\lambda]=1+\sum_{\vec{\nu}} \lambda_{\vec{\nu}}\left\langle\mathcal{O}_{\vec{\nu}}\right\rangle_{0}+\frac{1}{2} \sum_{\vec{\nu}_{1}, \vec{\nu}_{2}} \lambda_{\vec{\nu}_{1}} \lambda_{\vec{\nu}_{2}}\left\langle\mathcal{O}_{\vec{\nu}_{1}} \mathcal{O}_{\vec{\nu}_{2}}\right\rangle_{0}+\cdots
$$

The projection of $Z[\lambda]$ onto $\mathcal{O}_{\vec{\mu}}$ is straightforward at the sight of Eq. (3.33). The product of two Schur operators with $2 n_{1}$ and $2 n_{2}$ tensors is again a Schur operator

$$
\mathcal{O}_{\vec{\nu}_{1}} \mathcal{O}_{\vec{\nu}_{2}}=\sum_{\vec{\mu}} a_{\vec{\nu}_{1} \vec{\nu}_{2}}^{\vec{\mu}} \mathcal{O}_{\vec{\mu}}
$$

where $\mathcal{O}_{\vec{\mu}}$ is made of $2 n=2 n_{1}+2 n_{2}$ tensors and $a_{\vec{\nu}_{1} \vec{\nu}_{2}}^{\vec{\mu}}$ is proportional to the product of Littlewood-Richardson numbers $C_{\vec{\nu}_{1} \vec{\nu}_{2}}^{\vec{\mu}}$; see Appendix B. Higher order terms in the expansion are similar and involve coefficients $C_{\vec{\nu}_{1} \vec{\nu}_{2} \vec{\nu}_{3}}^{\overrightarrow{\overrightarrow{3}}}$, $C_{\vec{\nu}_{1} \vec{\nu}_{2} \vec{\nu}_{3} \vec{\nu}_{4}}^{\vec{\mu}}$, and so on. Thus, the natural projection of the expansion (3.33) onto $\mathcal{O}_{\vec{\mu}}$ reads

$$
\begin{aligned}
P_{\vec{\mu}}(Z[\lambda]) & =1+\lambda_{\vec{\mu}}\left\langle\mathcal{O}_{\vec{\mu}}\right\rangle_{0}+\sum_{\vec{\nu}_{1}, \vec{\nu}_{2}} \lambda_{\vec{\nu}_{1}} \lambda_{\vec{\nu}_{2}} a_{\vec{\nu}_{1} \vec{\nu}_{2}}^{\vec{\mu}}\left\langle\mathcal{O}_{\vec{\mu}}\right\rangle_{0}+\cdots \\
& =1+\left\langle\mathcal{O}_{\vec{\mu}}\right\rangle_{0}\left(\lambda_{\vec{\mu}}+\sum_{\vec{\nu}_{1}, \vec{\nu}_{2}} \lambda_{\vec{\nu}_{1}} \lambda_{\vec{\nu}_{2}} a_{\vec{\nu}_{1} \vec{\nu}_{2}}^{\vec{\mu}}+\cdots\right) .
\end{aligned}
$$

Similarly, we can Taylor expand Eq. (3.29) with respect to the coefficients of the higher derivative terms. For instance, in four dimensions, the most general quadratic, covariant, parity-invariant, metric-compatible, and torsion-free action is [43]

$$
\begin{aligned}
S_{F_{1}, F_{2}, F_{3}}[g]= & \int d^{4} x \frac{1}{2} \sqrt{g}\left(M_{p}^{2} R+R F_{1}(\square) R\right. \\
& \left.+R_{a b} F_{2}(\square) R^{a b}+R_{a b c d} F_{3}(\square) R^{a b c d}\right),
\end{aligned}
$$

with

$$
F_{i}(\square)=\sum_{n=0}^{\infty} f_{i_{n}} \frac{\square^{n}}{M^{2 n}}, \quad \square=g^{a b} \nabla_{a} \nabla_{b},
$$

where $M \leq M_{p}$ is a certain mass scale that cannot be too small so that the Einstein action is still accurate for the current observations. The proposed prescription for the gravity side is to first evaluate the action at the background $\mu$, and then perform the Taylor expansion of $e^{-S_{\kappa}[\vec{\mu}]}$ with respect to the coefficients $\frac{f_{i_{n}}}{M^{2 n}}$. The result can be reorganized as

$$
e^{-S_{\kappa}[\vec{\mu}]}=1+\gamma_{1} S_{1}[\vec{\mu}]+\gamma_{2} S_{2}[\vec{\mu}]+\cdots,
$$

where $S_{1}[\vec{\mu}]$ is the Hilbert-Einstein action evaluated at $\vec{\mu}$, $S_{2}[\vec{\mu}]$ will be the action of the terms quadratic in curvatures evaluated at $\vec{\mu}$, and so on. The coefficients $\gamma$ in Eq. (3.38) depend on products of the functions $f_{i}$ and on the scale. The dependence on the scale is

$$
\gamma_{1}=-\frac{M_{p}^{2}}{2}, \quad \gamma_{i} \sim M^{2-2 i}, \quad i \geq 2 .
$$

Now we equate Eq. (3.35) with Eq. (3.38). At leading order, we have

$$
\left\langle\mathcal{O}_{\vec{\mu}}\right\rangle_{0}=S_{H E}[\vec{\mu}] \text {. }
$$

\section{WARD IDENTITIES OF TENSOR MODELS AND LOCALITY}

Ward identities arise in tensor models associated with the change of integration variables in the partition function. That is with the change $T \rightarrow T+\delta T$ and $\bar{T} \rightarrow \bar{T}+\delta \bar{T}$ given by

$$
\begin{aligned}
T_{i_{1}, \ldots, i_{d}} & \rightarrow T_{i_{1}, \ldots, i_{d}}+\frac{\delta \mathcal{O}}{\delta T^{i_{1}, \ldots, i_{d}}}, \\
\bar{T}_{i_{1}, \ldots, i_{d}} & \rightarrow \bar{T}_{i_{1}, \ldots, i_{d}}+\frac{\delta \mathcal{O}}{\delta \bar{T}_{i_{1}, \ldots, i_{d}}},
\end{aligned}
$$

where $\mathcal{O}$ is a gauge-invariant operator. Using these transformations, it is found that the symmetries of the action translate into a tower of identities among averages; see 
$[14,44,45]$. For an action given by Eq. (3.27), these identities may be written as ${ }^{10}$

$$
N^{d-1}|\mathcal{O}|\langle\mathcal{O}\rangle=\sum_{\mathcal{O}^{\prime}} \lambda_{\mathcal{O}^{\prime}}\left\langle\left\{\mathcal{O}, \mathcal{O}^{\prime}\right\}\right\rangle+\langle\Delta \mathcal{O}\rangle,
$$

where $|\mathcal{O}|=n$ for an invariant made of $2 n$ tensors, the sum is over all invariants $\mathcal{O}^{\prime}$ present in the action (Schur invariants), and the averages are taken with the full action. I have denoted

$$
\begin{gathered}
\Delta \mathcal{O}=\frac{\delta^{2} \mathcal{O}[T]}{\delta T_{i_{1} \ldots i_{d}} \delta \bar{T}^{i_{1} \ldots i_{d}}}, \\
\left\{\mathcal{O}, \mathcal{O}^{\prime}\right\}=\frac{\delta \mathcal{O}[T]}{\delta T_{i_{1} \ldots i_{d}}} \frac{\delta \mathcal{O}^{\prime}[T]}{\delta \bar{T}^{i_{1} \ldots i_{d}}},
\end{gathered}
$$

which are the cut and join operators, respectively, defined in [14]. Similar operators, with different names, appear in the literature. For instance, in [1], these operators are called $\omega$ and $\Omega$, respectively.

For Gaussian averages, Eq. (4.2) turns into

$$
\langle\mathcal{O}\rangle_{0}=\frac{1}{n N^{d-1}}\langle\Delta \mathcal{O}\rangle_{0}
$$

We now apply Eq. (4.5) to Eq. (3.40) and obtain

$$
\frac{1}{n N^{d-1}}\left\langle\Delta \mathcal{O}_{\vec{\mu}}\right\rangle_{0}=S_{H E}[\vec{\mu}] .
$$

In the next subsection, I will show that an emergence of spacetime and a notion of locality are already present in Eq. (4.6).

\section{A. The spacetime grid}

It is proven in Appendix E that

$$
\begin{aligned}
\Delta \mathcal{O}_{\vec{\mu}} & =\sum_{\vec{\mu}^{\prime} \nearrow \vec{\mu}} C\left(\vec{\mu}, \vec{\mu}^{\prime}\right) \mathcal{O}_{\vec{\mu}^{\prime}}, \quad \text { with } \\
C\left(\vec{\mu}, \vec{\mu}^{\prime}\right) & =n^{3} \frac{g_{\vec{\mu}}}{g_{\vec{\mu}^{\prime} \uparrow \vec{\mu}}} \sum_{\vec{\mu}^{\prime} \nearrow \vec{\mu}} \frac{\operatorname{Dim}_{\vec{\mu}}(N)}{\operatorname{Dim}_{\vec{\mu}^{\prime}}(N)},
\end{aligned}
$$

where $\vec{\mu}^{\prime} \nearrow \vec{\mu}$ indicates the collection of $d$ partitions of $n-1$ elements that appear as we delete one of the corners of each of the $d$ partitions of $n$ elements $\vec{\mu}$. The are as many $\vec{\mu}^{\prime}$ 's in the expansion (4.7) as the product of the number of corners of the $d$ partitions in $\vec{\mu}$. Since each partition $\mu_{i}$ has at most $N$ parts, its number of corners is at most equal to $N$. Let us associate with each corner $c_{j}\left(\mu_{i}\right)$ the number of the row it appears at. For instance,

\footnotetext{
${ }^{10}$ See [45], Eq. (2.2).
}

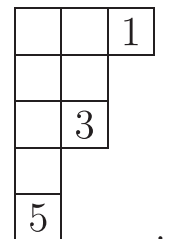

With this association every $\vec{\mu}^{\prime}$ of $\vec{\mu}^{\prime} \nearrow \vec{\mu}$ is mapped to a $d$-tuple made by choosing the value of the deleted corner in each diagram $\mu_{i}$. Let us see an example. Consider, for $d=3$ and $n=5$,

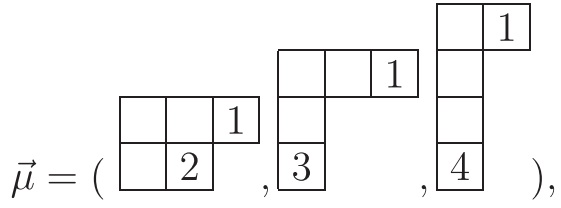

where we have already labeled the corners. Then one of the subduced $\vec{\mu}^{\prime}$ 's is

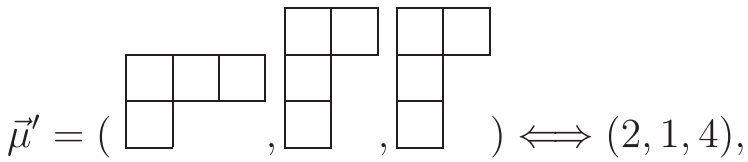

and another is

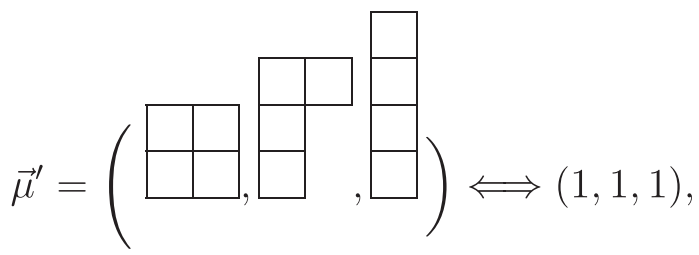

Let us consider a $N^{d}$ grid parametrized by $\vec{r}$ with $r_{i}=1, \ldots, N$. With the above prescription, each $\vec{\mu}^{\prime}$ can be mapped to a point in the grid

$$
\vec{\mu}^{\prime} \nearrow \vec{\mu} \mapsto \vec{r}
$$

Since the number of corners of each $\mu_{i}$ is always less than or equal to $N$, in general, not all the points in the grid get occupied, but the map is injective. Thus, given a label $\vec{\mu}$, there is no point in the grid that is mapped to two different $\vec{\mu}^{\prime}$ 's. Now with this map any sum $\sum_{\vec{\mu}^{\prime}} \nearrow_{\vec{\mu}}$, and specifically Eq. (4.7), can be understood as a sum over the grid. For that, the function $C\left(\vec{\mu}, \vec{\mu}^{\prime}\right)$ must be interpolated so that it takes values all over the grid. On the rhs of Eq. (4.6), we have a sum over the entire volume of spacetime which, by splitting each coordinate into $N$ parts, can be discretized and turned into the sum 


$$
\int_{V} d^{d} x R \sqrt{g} \rightarrow \sum_{\vec{r} \in \text { grid }} R(\vec{r}) .
$$

Equation (4.6) turns into an equality between two sums over the grid. The equation is automatically fulfilled if we identify the summands on both sides of the equation. In this case, we obtain

$$
\frac{1}{n N^{d-1}} C(\vec{r})\left\langle\mathcal{O}_{\vec{r}}\right\rangle_{0}=R(\vec{r}),
$$

where, on the lhs, we have already performed the map $\vec{\mu}^{\prime} \rightarrow \vec{r}$. Equation (4.14) provides a prescription for the emergence of a background in tensor models.

\section{B. Locality}

One of the nice features of the picture proposed in Eq. (4.14) is the emergence of a sense of locality in tensor models. Nearby points in the grid correspond to nearby corners in the Young diagrams $\vec{\mu}$. The quantity on the lhs of Eq. (4.14) varies from one point to the grid to another according to the "corner distance" in the Young diagrams, which for adjacent corners is minimal. It will be infinitesimal when we take the continuum limit. If we want to reconstruct a background from a tensor model, this fact translates, via Eq. (4.14), into a smooth curvature and thus a regular manifold. In short, the main message of Eq. (4.14) is the fact that the sense of locality in tensor models is related to nearby corners in the Young diagrams which label Schur invariants. A similar idea of locality was already suggested in the context of holography in [46] and recently developed in [47].

\section{ADM mass}

For tensor models with matter, we have a variety of backgrounds. Regular manifolds, with nonsingular curvature, will relate to balanced Young diagrams. Remember that balanced diagrams, or limit shapes, are the ones which label regular irreducible representations of $S_{\infty}$ [26]. Those diagrams can be constructed inductively by adding boxes and rescaling, in a way such that in the limit $n \rightarrow \infty$ we obtain a monotonic function enclosing a nonzero area with the axis. Thus, $\vec{\mu} \rightarrow\left(f_{1}, \ldots, f_{d}\right)$. The nonzero area condition, together with the fact that no Young diagram exceeds $N$ rows, leads to the conclusion that $n \sim N^{2}$, which is also the relation found in holography for the states that relate to new geometries. Thus, in the classical limit with $N, n \rightarrow \infty$, the relevant quantity will be

$$
\rho=\frac{n}{N^{2}} \in \mathbb{R}_{+},
$$

and we expect all nonzero nondivergent classical quantities to depend on $n$ and $N$ only through $\rho$.
Let us consider asymptotically flat spacetimes. It is known that those spaces admit an ADM mass, that is, a masslike charge associated with the entire spacetime. It is natural to wonder what this charge is in the tensorial description and whether or not we can read it off from the labels of Schur operators.

The positive number $\rho$ is an intrinsic quantity of Schur operators and ranges from 0 to $\infty$. It is related to the energy of the state produced as the Schur operator acts on the vacuum state. Indeed, the energy of the Schur states must grow linearly with $n$, as it does for the harmonic oscillator, ${ }^{11}$ recovering the vacuum for $n=0$. These considerations make $\rho$ a good candidate for the ADM mass of the associated geometry. Thus, I propose that the ADM mass be given by the quantity

$$
M_{\mathrm{ADM}}=C \rho,
$$

where $C$ does not depend on either $n$ or $N$. Note that for $n=0$ we have a flat spacetime. Using Eq. (4.16) and suitable configurations $\vec{\mu}$ compatible with black hole geometries (likely based on symmetry arguments), it would be interesting to compute the Bekenstein-Hawking entropy and compare it to the direct counting of (tensor) microstates. This would provide a valuable check of this proposal. The task does not seem easy, however, since it involves counting the number of invariants $\mathcal{O}_{\vec{\mu}, i j}$ compatible with the symmetries that, in the end, translates into counting sums of Kronecker coefficients. I will consider this in a future work.

\section{SUMMARY AND OUTLOOK}

In this paper I have provided a picture of emergent spacetime by making a connection between tensor models at the large $N$ and $n$ limit and classical gravity. In contrast to the usual correspondence between permutation invariants and triangulations, in this proposal Schur invariants take a prominent role and are mapped to backgrounds. At the heart of this proposal is the reorganization of the space of invariants into a basis driven by representation theory, the restricted Schur basis, which is found to be suitable for the connection with gravity at the large $N$ and $n$ limit.

Without a background it is hard to make any sense of locality. Thus, it is always challenging for a background independent theory of gravity to incorporate local theories which, at the end of the day, are the ones that describe most of the physics phenomena we are able to test. In my

\footnotetext{
${ }^{11}$ One can take the analogy with the harmonic oscillator further. The tensor model can be understood as a collection of $N^{d}$ identical harmonic oscillators, one for each component of the tensor. Then each component acts as the creation operator and its conjugate as the annihilation operator when acting on the vacuum state. With this picture, an invariant made of $n$ tensors acting on the vacuum produces a sum of excited states, all with excitation number $d n$.
} 
proposal, by the use of Ward identities, I am able to offer a natural sense of locality in the tensor invariant which encode backgrounds: two points in the grid are close if the corners of the Young diagrams they are mapped to are close. This way, the corner distance in the Young diagrams that label the Schur invariants in the tensor model translates into physical distance in gravity.

There are a number of lines I would find interesting to explore in future works. The correspondence between tensorial and gravitational quantities is still incomplete. More examples will provide a more detailed picture of the interrelation between the two theories. For instance, it would be very interesting to reproduce the black hole entropy in the tensor picture by counting the tensor states compatible with the Schwarzschild geometry. Besides, the tensor theory (and the full gravity theory) are not determined in this paper since the couplings of the different interaction terms in the action are not fixed. They could be fixed by a sensible renormalization flow equation [48]. Interestingly, a Wetterich type equation has already been proposed in tensor models [39], where the sector corresponding to gravity sits at a fixed point of the flow. This way, universality is expected to wipe out all the spurious details of the discretizations.

\section{ACKNOWLEDGMENTS}

I would like to thank Robert de Mello Koch for his valuable feedback, encouragement, and support regarding both conceptual and technical aspects of the paper. This work was supported by Universidad de Zaragoza.

\section{APPENDIX A: RESTRICTED SCHUR BASIS AND SCHUR INVARIANTS}

\section{Schur invariants}

Since the observables are built on $n$ indistinguishable copies of $T$ and $\bar{T}$, it is straightforward to see that operators in Eq. (2.3) enjoy the symmetry of shuffling the copies of $T$ and $\bar{T}$ independently, so one must consider

$$
\mathcal{O}_{\vec{\alpha}} \sim \mathcal{O}_{\sigma \cdot \vec{\alpha} \cdot \tau}, \quad \sigma, \tau \in S_{n} .
$$

A generic observable is a linear combination of generators $\mathcal{O}_{\vec{\alpha}}$, so it can be written as

$$
\mathcal{O}_{f}=\sum_{\vec{\alpha}} f(\vec{\alpha}) \mathcal{O}_{\vec{\alpha}},
$$

where $f: S_{n}^{d} \rightarrow \mathbb{C}$.

Owing to the symmetry (A1), a basis of observables is given by a basis of complex functions $f(\vec{\alpha})$ with the property

$$
f(\sigma \cdot \vec{\alpha} \cdot \tau)=f(\vec{\alpha}), \quad \sigma, \tau \in S_{n},
$$

which will be called from now on double coset invariant (DCI) functions.

Note that the problem is analogous to finding class functions of a symmetric group, functions with the property $\chi\left(\sigma \alpha \sigma^{-1}\right)=\chi(\alpha)$. The last functions are the well-known characters of the symmetric group. The task, in order to obtain a basis of observables, is therefore to find a basis of double coset functions.

The usual convolution algebra of functions of the symmetric group can be extended to the double coset invariant functions as

$$
h(\vec{\alpha})=f * g(\vec{\alpha})=\sum_{\vec{\beta} \in S_{n}^{d}} f(\vec{\beta}) g\left(\vec{\beta}^{-1} \cdot \vec{\alpha}\right) .
$$

It is easy to see that $h(\sigma \vec{\alpha} \tau)=h(\vec{\alpha})$, so Eq. (A4) defines an algebra of double coset functions. This algebra is noncommutative, but it is associative and it has the unit function

$$
f * \delta_{\mathrm{DCI}}(\vec{\alpha})=\delta_{\mathrm{DCI}} * f(\vec{\alpha})=f(\vec{\alpha}) .
$$

The unit element $\delta_{\mathrm{DCI}}$ is constructed by means of the delta function of the symmetric group $\delta(\sigma)$, which is 0 unless $\sigma$ is the identity, in which case it is 1 . Thus,

$$
\delta_{\mathrm{DCI}}(\vec{\alpha})=\sum_{\sigma \in S_{n}} \delta(\vec{\alpha} \cdot \sigma),
$$

which is double coset invariant, and it is 0 unless $\vec{\alpha}=(\tau, \ldots, \tau)$ for any $\tau \in S_{n}$. Using the identity

$$
\delta(\sigma)=\frac{1}{n !} \sum_{\mu \vdash n} d_{\mu} \chi_{\mu}(\sigma),
$$

we can also set the unit element $\delta_{\mathrm{DCI}}(\vec{\alpha})$ in terms of characters of the symmetric group as

$$
\delta_{\mathrm{DCI}}(\vec{\alpha})=\frac{1}{n !^{d}} \sum_{\vec{\mu}} \sum_{\sigma \in S_{n}} d_{\vec{\mu}} \chi_{\vec{\mu}}(\vec{\alpha} \cdot \sigma) .
$$

The expansion of the unit function (A8) indicates that the function defined as

$$
\mathcal{P}_{\vec{\mu}}(\vec{\alpha})=\frac{1}{n !^{d}} \sum_{\sigma \in S_{n}} d_{\vec{\mu}} \chi_{\vec{\mu}}(\vec{\alpha} \cdot \sigma)
$$

projects onto the subspace of operators labeled $\vec{\mu}$.

It is easy to see that the Schur invariants $\mathcal{O}_{\vec{\mu}}$ are driven by projectors. That is,

$$
\mathcal{O}_{\vec{\mu}}=\sum_{i=1}^{g_{\vec{\mu}}} \mathcal{O}_{\vec{\mu} ; i i}=\sum_{\vec{\alpha}} \mathcal{P}_{\vec{\mu}}(\vec{\alpha}) \mathcal{O}_{\vec{\alpha}} .
$$


Schur invariants form a distinguished sector of the restricted Schur basis and play a predominant role in this paper as partners of backgrounds.

\section{Restricted Schur basis and correlators}

With the Schur basis of DCI functions

$I=\left\{F_{\vec{\mu} ; i j}(\alpha) \mid \quad \mu_{i} \vdash n, \quad l\left(\mu_{i}\right) \leq N, \quad i, j=1, \ldots, g_{\vec{\mu}}\right\}$

the convolution algebra (A4) can be written as

$$
F_{\vec{\mu} ; i j} * F_{\vec{\nu} ; k l}(\vec{\alpha})=\delta_{\vec{\mu} \vec{\nu}} \delta_{j k} F_{\vec{\mu} ; i l}(\vec{\alpha}) .
$$

Since DCI fucntions act naturally on trace operators to produce operator invariants, the algebra (A12) induces an algebra in the space of invariant operators. The algebra (A12) is also compatible with the involution

$$
\bar{F}_{\vec{\mu} ; i j}(\vec{\alpha})=F_{\vec{\mu} ; j i}\left(\vec{\alpha}^{-1}\right) .
$$

Let us define the matrix $\left(\mathcal{M}_{\vec{\mu}}(\vec{\alpha})\right)_{i j}$ of size $g_{\vec{\mu}} \times g_{\vec{\mu}}$ which contains the function $F_{\vec{\mu} ; i j}(\vec{\alpha})$ at the site $(i j)$. Note that a unitary transformation with $U\left(g_{\vec{\mu}}\right)$ acting on $\mathcal{M}_{\vec{\mu}}(\vec{\alpha})$ as $U\left(g_{\vec{\mu}}\right)_{i j}\left(\mathcal{M}_{\vec{\mu}}(\vec{\alpha})\right)_{j k} U^{-1}\left(g_{\vec{\mu}}\right)_{k l}$ does not alter the convolution structure (A12). In view of Eq. (A13) it is easy to see that $\mathcal{M}_{\vec{\mu}}(\overrightarrow{1})$ is self-adjoint. This means that by rearranging the basis with a unitary transformation we can make $\mathcal{M}_{\vec{\mu}}(\overrightarrow{1})$ diagonal, that is, $F_{\vec{\mu} ; j i}(\overrightarrow{1}) \propto \delta_{i j}$. The proportionality constant must be consistent with the normalization of the invariants $\mathcal{O}_{\vec{\mu}}$, defined in Eq. (2.23), and their Gaussian averages (2.30). They read

$$
F_{\vec{\mu} ; j i}(\overrightarrow{1})=\delta_{i j} \frac{d_{\vec{\mu}}}{n !^{d-1}} .
$$

Thus, our basis of DCI functions will fulfill (A14). A basis of invariant operators $\left\{\mathcal{O}_{\vec{\mu}, i j}\right\}$ is obtained by acting with each element of Eq. (A11) on trace invariants as

$$
\mathcal{O}_{\vec{\mu}, i j}=\sum_{\vec{\alpha}} F_{\vec{\mu} ; j i}(\vec{\alpha}) \mathcal{O}_{\vec{\alpha}}
$$

Now let us compute the expectation value of the elements of the basis (A11). Using Eq. (2.28), we find that the Gaussian averages of the elements of the restricted Schur basis read

$$
\begin{aligned}
\left\langle\mathcal{O}_{\vec{\mu} ; i j}\right\rangle_{0} & =\sum_{\vec{\alpha}} F_{\vec{\mu} ; i j}(\vec{\alpha})\left\langle\mathcal{O}_{\vec{\alpha}}\right\rangle_{0} \\
& =\frac{1}{N^{n(d-1)}} \sum_{\vec{\alpha}} \sum_{\sigma \in S_{n}} F_{\vec{\mu} ; i j}(\vec{\alpha}) N^{C(\vec{\alpha} \cdot \sigma)} \\
& =\frac{1}{N^{n(d-1)}} \sum_{\vec{\alpha}, \vec{\nu}} \sum_{\sigma \in S_{n}} F_{\vec{\mu} ; i j}(\vec{\alpha}) \chi_{\vec{\nu}}(\vec{\alpha} \cdot \sigma) \operatorname{Dim}_{\vec{\nu}}(N) \\
& =\frac{n !^{d}}{N^{n(d-1)}} \sum_{\vec{\nu}, \vec{\alpha}} F_{\vec{\mu} ; i j}(\vec{\alpha})\left[\frac{1}{n ! d} \sum_{\sigma} d_{\vec{\nu}} \chi_{\vec{\nu}}(\vec{\alpha} \cdot \sigma)\right] \frac{\operatorname{Dim}_{\vec{\nu}}(N)}{d_{\vec{\nu}}} \\
& =\frac{n !^{d}}{N^{n(d-1)}} \sum_{\vec{\nu}, \vec{\alpha}} F_{\vec{\mu} ; i j}(\vec{\alpha}) \mathcal{P}_{\vec{\nu}}(\vec{\alpha}) \frac{\operatorname{Dim}_{\vec{\nu}}(N)}{d_{\vec{\nu}}} \\
& =\frac{n !^{d}}{N^{n(d-1)}} F_{\vec{\mu} ; i j}(\overrightarrow{1}) \frac{\operatorname{Dim}_{\vec{\mu}}(N)}{d_{\vec{\mu}}} \\
& =\delta_{i j} \frac{n !}{N^{n(d-1)}} \operatorname{Dim}_{\vec{\mu}}(N),
\end{aligned}
$$

which is consistent with the averages of Shur invariants (2.30) and proves that the constant of proportionality in Eq. (A14) was chosen appropriately.

\section{APPENDIX B: PRODUCT OF TWO SCHUR OPERATORS}

The product of two Schur operators, one with $2 n$ tensors and the other with $2 m$ tensors, is

$$
\begin{aligned}
\mathcal{O}_{\vec{\mu}} \mathcal{O}_{\vec{\nu}}= & \frac{1}{(n ! m !)^{d-1}} \sum_{\vec{\alpha} \in S_{n}^{d}} \sum_{\vec{\beta} \in S_{m}^{d}} d_{\vec{\mu}} d_{\vec{\nu}} \chi_{\vec{\mu}}(\vec{\alpha}) \chi_{\vec{\nu}}(\vec{\beta}) \mathcal{O}_{\vec{\alpha}} \mathcal{O}_{\vec{\beta}} \\
= & \frac{d_{\vec{\mu}} d_{\vec{\nu}}}{(n ! m !)^{d-1}} \sum_{\vec{\alpha} \in S_{n}^{d}} \sum_{\vec{\beta} \in S_{m}^{d}} \chi_{\vec{\mu}}(\vec{\alpha}) \chi_{\vec{\nu}}(\vec{\beta}) \mathcal{O}_{\vec{\alpha} \circ \vec{\beta}} \\
= & \frac{d_{\vec{\mu}} d_{\vec{\nu}}}{(n ! m !)^{d-1}} \sum_{\vec{\alpha} \in S_{n}^{d}} \sum_{\vec{\beta} \in S_{m}^{d}} \chi_{\vec{\mu}}(\vec{\alpha}) \chi_{\vec{\nu}}(\vec{\beta}) \\
& \times \sum_{\lambda_{i} \vdash n+m} \frac{1}{(n+m) !^{d}} \chi_{\vec{\lambda}}(\vec{\alpha} \circ \vec{\beta}) \chi_{\vec{\lambda}}(\vec{\rho}) \mathcal{O}_{\vec{\rho}} \\
= & \frac{d_{\vec{\mu}} d_{\vec{\nu}}}{(n ! m !)^{d-1}} \sum_{\vec{\alpha} \in S_{n}^{d}} \sum_{\vec{\beta} \in S_{m}^{d}} \chi_{\vec{\mu}}(\vec{\alpha}) \chi_{\vec{\nu}}(\vec{\beta}) \\
& \times \sum_{\lambda_{i} \vdash n+m} \frac{1}{(n+m) ! d_{\vec{\lambda}}} \chi_{\vec{\lambda}}(\vec{\alpha} \circ \vec{\beta}) \mathcal{O}_{\vec{\lambda}} \\
= & \frac{n ! m !}{(n+m) !} \sum_{\lambda_{i} \vdash n+m} \frac{d_{\vec{\mu}} d_{\vec{\nu}}}{d_{\vec{\lambda}}} C_{\vec{\mu} \vec{\nu}} \mathcal{O}_{\vec{\lambda}},
\end{aligned}
$$

where we have used the Littlewood-Richardson coefficients and the products of them defined in Eqs. (2.8) and (2.9). 


\section{APPENDIX C: CASIMIR OPERATORS ACTING ON IRREDUCIBLE REPRESENTATIONS}

In order to compute the action of cut operators on Schur invariants $\mathcal{O}_{\vec{\mu}}$, it is necessary to know how certain Casimir operators of the group algebra $\mathbb{C}\left(S_{n}\right)$ act on representations. It is known that a Casimir operator ${ }^{12}$ acting on an irreducible representation of $S_{n}$ results in a multiple of the identity of that irreducible representation. That is,

$$
\Gamma_{\mu}(\mathcal{C} \sigma)=C(\mu) \Gamma_{\mu}(\sigma), \quad \mu \vdash n, \quad \sigma \in S_{n},
$$

where $\mathcal{C}$ is a Casimir of $\mathbb{C}\left(S_{n}\right)$ and $C(\mu)$ is a number. Let us see some examples. Consider the Casimir $\mathbb{C}\left(S_{n}\right)$ built as a sum of all transpositions,

$$
\mathcal{T}_{2}(n)=\sum_{i<j}^{n}(i j)
$$

Now, according to $(\mathrm{C} 1)$,

$$
\Gamma_{\mu}\left(\mathcal{T}_{2}(n) \sigma\right)=T_{2}(\mu) \Gamma_{\mu}(\sigma), \quad \mu \vdash n, \quad \sigma \in S_{n} .
$$

In order to find out the value of $T_{2}(\mu)$, we make $\sigma=1$ and take traces in Eq. (C3). So,

$$
\chi_{\mu}\left(\mathcal{T}_{2}(n)\right)=T_{2}(\mu) \chi_{\mu}(1)=T_{2}(\mu) d_{\mu} .
$$

The lhs of Eq. (C4) is the sum of $\left(\begin{array}{l}n \\ 2\end{array}\right)$ characters of $\Gamma_{\mu}$ evaluated on a transposition. Using the MurnaghanNakayama rule, it is easy to find that the character of any two-cycle element is

$$
\chi_{\mu}((i j))=\frac{d_{\mu}}{\left(\begin{array}{l}
n \\
2
\end{array}\right)} \sum_{i=1}^{l(\mu)}\left(\frac{\mu_{i}\left(\mu_{i}-1\right)}{2}-\frac{\mu_{i}^{t}\left(\mu_{i}^{t}-1\right)}{2}\right)
$$

where $\mu_{i}$ is the length of row $i$ and the superscript $t$ indicates the transposed diagram. Inserting Eq. (C5) into Eq. (C4), we find that

$$
T_{2}(\mu)=\sum_{i=1}^{l(\mu)}\left(\frac{\mu_{i}\left(\mu_{i}-1\right)}{2}-\frac{\mu_{i}^{t}\left(\mu_{i}^{t}-1\right)}{2}\right) .
$$

For our purposes it will be useful to find the action of the Jucys-Murphy element

$$
\mathcal{J}_{n}=(n 1)+(n 2)+\ldots(n n-1),
$$

which is a Casimir of $\mathbb{C}\left(S_{n-1}\right)$ onto irreducible representations of $S_{n-1}$. Specifically, we will need to compute $\chi_{\mu}\left(\sigma^{\prime} \mathcal{J}_{n}\right)$, where $\mu \vdash n$ but $\sigma^{\prime} \in S_{n-1}$. First, we realize

\footnotetext{
${ }^{12}$ Casimir operators are elements which belong to the center of $\mathbb{C}\left(S_{n}\right)$; that is, they commute with every element of $\mathbb{C}\left(S_{n}\right)$.
}

that the Jucys-Murphy element is a sum of two Casimir operators

$$
\mathcal{J}_{n}=\mathcal{T}_{2}(n)-\mathcal{T}_{2}(n-1) .
$$

Both $\mathcal{T}_{2}(n)$ and $\mathcal{T}_{2}(n-1)$ commute with all the elements of $\mathbb{C}\left(S_{n-1}\right)$. We compute

$$
\chi_{\mu}\left(\mathcal{J}_{n} \sigma^{\prime}\right)=\chi_{\mu}\left(\mathcal{T}_{2}(n) \sigma^{\prime}\right)-\chi_{\mu}\left(\mathcal{T}_{2}(n-1) \sigma^{\prime}\right) .
$$

Remember that if $\sigma^{\prime} \in S_{n-1}$,

$$
\Gamma_{\mu}\left(\sigma^{\prime}\right)=\bigoplus_{\mu^{\prime} \nearrow \mu} \Gamma_{\mu^{\prime}}\left(\sigma^{\prime}\right) .
$$

The operator $\mathcal{T}_{2}(n-1)$ acts on each irreducible representation of the direct sum. Applying Eq. (C6), we obtain

$$
\chi_{\mu}\left(\mathcal{J}_{n} \sigma^{\prime}\right)=\sum_{\mu^{\prime} \nearrow^{\mu}} J\left(\mu, \mu^{\prime}\right) \chi_{\mu^{\prime}}\left(\sigma^{\prime}\right),
$$

where $J\left(\mu, \mu^{\prime}\right)$ is the content of the (corner) box which must be deleted from diagram $\mu$ to obtain diagram $\mu^{\prime}$. The content of the box in position $(i, j)$ (that is, the box at row $i$ and column $j$ ) is simply $j-i$. An example of a Young diagram where the content of the boxes have been spelled out is

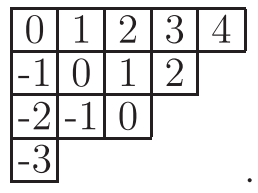

\section{APPENDIX D: CASIMIR OPERATORS ON THE DIAGONAL ACTION}

With the notation we have been using, a diagonal action on a product of irreducible representations is defined as

$$
\sigma \cdot \Gamma_{\vec{\mu}}(\vec{\alpha})=\Gamma_{\vec{\mu}}(\vec{\alpha} \cdot \sigma) .
$$

As usual, when an action is defined on a vector space it automatically splits the space into subspaces, which are irreducible representations of the group. The quantities that appear in this paper involve sums like

$$
\sum_{\sigma \in S_{n}} \Gamma_{\vec{\mu}}(\vec{\alpha} \cdot \sigma) .
$$

The object (D2) is indeed an irreducible representation of the diagonal action; specifically, it is the symmetric representation $(n)$ of the diagonal action. As in the case of nondiagonal actions, a Casimir operator acting on it will result in a multiple of the identity. For instance, 


$$
\sum_{\sigma \in S_{n}} \Gamma_{\vec{\mu}}\left(\vec{\alpha} \cdot \sigma \mathcal{T}_{2}(n)\right)=T(\vec{\mu}) \sum_{\sigma \in S_{n}} \Gamma_{\vec{\mu}}(\vec{\alpha} \cdot \sigma)
$$

Taking traces and $\vec{\alpha}=\overrightarrow{1}$, we see that

$$
\sum_{\sigma \in S_{n}} \chi_{\vec{\mu}}\left(\sigma \mathcal{T}_{2}(n)\right)=T(\vec{\mu}) \sum_{\sigma \in S_{n}} \chi_{\vec{\mu}}(\sigma)
$$

from which we find that

$$
T(\vec{\mu})=\left(\begin{array}{l}
n \\
2
\end{array}\right)
$$

In this paper we need to compute the more involved quantity $\sum_{\sigma^{\prime} \in S_{n-1}} \Gamma_{\vec{\mu}}\left(\sigma^{\prime} \mathcal{J}_{n}\right)$. As before, we will be using the fact that

$$
\Gamma_{\vec{\mu}}\left(\sigma^{\prime}\right)=\bigoplus_{\vec{\mu} \nearrow^{\prime}} \Gamma_{\vec{\mu}^{\prime}}\left(\sigma^{\prime}\right)
$$

First, note that, by simply splitting the terms in the sum, we have

$$
\sum_{\sigma^{\prime} \in S_{n-1}} \Gamma_{\vec{\mu}}\left(\mathcal{J}_{n} \sigma^{\prime}\right)=\sum_{\sigma \in S_{n}} \Gamma_{\vec{\mu}}(\sigma)-\sum_{\sigma^{\prime} \in S_{n-1}} \Gamma_{\vec{\mu}}\left(\sigma^{\prime}\right) .
$$

Now, since $\mathcal{J}_{n}$ is a Casimir of $\mathbb{C}\left(S_{n-1}\right)$, its action on an irreducible representation is proportional to the identity, so

$$
\sum_{\sigma^{\prime} \in S_{n-1}} \Gamma_{\vec{\mu}}\left(\mathcal{J}_{n} \sigma^{\prime}\right)=J(\vec{\mu}) \sum_{\sigma^{\prime} \in S_{n-1}} \Gamma_{\vec{\mu}}\left(\sigma^{\prime}\right) .
$$

Taking traces in Eqs. (D7) and (D8), we find that

$$
(J(\vec{\mu})+1) \sum_{\sigma^{\prime} \in S_{n-1}} \chi_{\vec{\mu}}\left(\sigma^{\prime}\right)=\sum_{\sigma \in S_{n}} \chi_{\vec{\mu}}(\sigma) .
$$

Let us call

$$
g_{\vec{\mu}^{\prime} \uparrow \vec{\mu}} \equiv \sum_{\vec{\mu}^{\prime} \nearrow \vec{\mu}} g_{\vec{\mu}^{\prime}}=\frac{1}{(n-1) !} \sum_{\sigma^{\prime} \in S_{n-1}} \chi_{\vec{\mu}}\left(\sigma^{\prime}\right) .
$$

Then

$$
\sum_{\sigma^{\prime} \in S_{n-1}} \chi_{\vec{\mu}}\left(\sigma^{\prime}\right)=\sum_{\substack{\sigma^{\prime} \in S_{n-1} \\ \vec{\mu}^{\prime}>\vec{\mu}}} \chi_{\vec{\mu}^{\prime}}\left(\sigma^{\prime}\right)=(n-1) ! g_{\vec{\mu}} \uparrow \vec{\mu}
$$

and

$$
J(\vec{\mu})=n \frac{g_{\vec{\mu}}}{g_{\vec{\mu}} \uparrow \vec{\mu}}-1 .
$$

It is easy to prove that Eq. (D12) agrees with the prescription given above for the case of only one representation,

$$
\sum_{\sigma^{\prime} \in S_{n-1}} \Gamma_{\mu}\left(\mathcal{J}_{n} \sigma^{\prime}\right)=J(\mu) \sum_{\sigma^{\prime} \in S_{n-1}} \Gamma_{\mu}\left(\sigma^{\prime}\right),
$$

where $g_{\mu}=\delta_{\mu(n)}$ and $g_{\mu^{\prime} \uparrow \mu}=1$. In this case

$$
J(\mu=(n))=n-1, \quad J(\mu=(n-1,1))=-1,
$$

and zero in the rest of the cases, as the prescription of the content of the boxes indicates. Using Eq. (D12), we see from Eq. (D7) that

$$
\sum_{\sigma \in S_{n}} \Gamma_{\vec{\mu}}(\sigma)=n \frac{g_{\vec{\mu}}}{g_{\vec{\mu}{ }^{\prime} \uparrow \vec{\mu}}} \sum_{\sigma^{\prime} \in S_{n-1}} \Gamma_{\vec{\mu}}\left(\sigma^{\prime}\right),
$$

which is the result that we are using in Appendix E.

\section{APPENDIX E: CUT OPERATORS ACTING ON SCHUR INVARIANTS}

The cut operator defined in [14] reads

$$
\Delta \mathcal{O}=\frac{\delta^{2} \mathcal{O}[T]}{\delta T_{i_{1} \ldots i_{d}} \delta \bar{T}^{i_{1} \ldots i_{d}}} .
$$

In this Appendix, to simplify notation, we are going to consider tensors with three indices, what means that $d=3$ in what follows. The general case can be straightforwardly recovered. The cut operator acts on Schur invariants as

$$
\begin{aligned}
& \Delta \mathcal{O}_{\vec{\mu}}=\frac{1}{n !^{d}} \sum_{\substack{r, s=1 \\
\alpha, \alpha_{2}, \alpha_{3}, \sigma \in S_{n}}}^{n} d_{\vec{\mu}} \chi_{\vec{\mu}}(\vec{\alpha} \cdot \sigma) \delta_{i_{r}}^{i_{\alpha_{1}(s)}} \delta_{j_{r}}^{j_{\alpha_{2}(s)}} \delta_{k_{r}}^{k_{\alpha_{3}(s)}} \\
& \times T_{i_{1} j_{1} k_{1}} \underset{r}{\cdots} T_{i_{n} j_{n} k_{n}} \bar{T}^{i_{\alpha_{1}(1)} j_{\alpha_{2}(1)} k_{\alpha_{3}(1)}} \underset{\hat{s}^{\prime}}{\ldots} \bar{T}^{i_{\alpha(n)} j_{\alpha_{2}(n)} k_{\alpha_{3}(n)}} .
\end{aligned}
$$

Performing the changes

$$
\tilde{\alpha}_{i}=\left(\begin{array}{ll}
n & r
\end{array}\right) \alpha_{i}\left(\begin{array}{ll}
n & s
\end{array}\right),
$$

taking into account that

$$
\sum_{\sigma \in S_{n}} \chi_{\vec{\mu}}(\overrightarrow{\tilde{\alpha}} \cdot \sigma)=\sum_{\sigma \in S_{n}} \chi_{\vec{\mu}}(\vec{\alpha} \cdot \sigma),
$$

and relabeling the permutations, we arrive at 


$$
\Delta \mathcal{O}_{\vec{\mu}}=\frac{n^{2}}{n !} \sum_{\vec{\alpha}, \sigma} d_{\vec{\mu}} \chi_{\vec{\mu}}(\vec{\alpha} \cdot \sigma) \delta_{i_{n}}^{i_{\alpha_{1}(n)}} \delta_{j_{n}}^{j_{\alpha_{2}(n)}} \delta_{k_{n}}^{k_{\alpha_{3}(n)}} T_{i_{1} j_{1} k_{1}} \cdots \times T_{i_{n-1} j_{n-1} k_{n-1}} \bar{T}^{i_{\alpha_{1}(1)} j_{\alpha_{2}(1)}} k_{\alpha_{3}(1)} \cdots \bar{T}^{i_{\alpha_{1}(n-1)} j_{\alpha_{2}(n-1)}} k_{\alpha_{3}(n-1)}
$$

First, we apply the result (D15) in the Appendix. The diagonal sum over $\sigma \in S_{n}$ turns into a sum over $\sigma^{\prime} \in S_{n-1}$, producing a global factor. We obtain

$$
\Delta \mathcal{O}_{\vec{\mu}}=\frac{n^{3}}{n !} \frac{g_{\vec{\mu}}}{g_{\vec{\mu}{ }^{\prime} \uparrow \vec{\mu}}} \sum_{\vec{\alpha}, \sigma^{\prime}} d_{\vec{\mu}} \chi_{\vec{\mu}}\left(\vec{\alpha} \cdot \sigma^{\prime}\right) \delta_{i_{n}}^{i_{\alpha_{1}(n)}} \delta_{j_{n}}^{j_{\alpha_{2}(n)}} \delta_{k_{n}}^{k_{\alpha_{3}(n)}} \times T_{i_{1} j_{1} k_{1}} \cdots T_{i_{n-1} j_{n-1} k_{n-1}} \bar{T}^{i_{\alpha_{1}(1)} j_{\alpha_{2}(1)} k_{\alpha_{3}(1)}} \cdots \bar{T}^{i_{\alpha_{1}(n-1)} j_{\alpha_{2}(n-1)} k_{\alpha_{3}(n-1)}} .
$$

The next step is to write the elements $\alpha_{1}, \alpha_{2}, \alpha_{3} \in S_{n}$ as elements of $S_{n-1}$ composed with a transposition. Note that the decomposition

$$
\alpha=(s n) \alpha^{\prime}, \quad \alpha \in S_{n}, \quad \alpha^{\prime} \in S_{n-1}, \quad s=1, \ldots, n,
$$

where $\alpha^{\prime}$ does not involve $n$, is unique. All permutations $\alpha \in S_{n}$ are obtained without repetition as we run over $\alpha^{\prime} \in S_{n-1}$ and $s=1, \ldots, n$ in Eq. (E7). With the parametrization (E7) $\alpha(n)=s$, and $\alpha(n)=n$ only when $s=n$. Besides, if written in disjoint cycles notation, the permutation $\alpha^{\prime}$ is obtained from $\alpha$ by simply deleting the "letter" $n$.

Now let us decompose each sum over $\alpha_{i}$ in Eq. (E6) into the sum over $\alpha_{i}^{\prime}$ and the sum over $\mathcal{J}_{n} \alpha_{i}^{\prime}$. This splitting has a purpose. Note that

$$
\delta_{i_{n}}^{i_{\alpha_{1}(n)}} \delta_{j_{n}}^{j_{\alpha_{2}(n)}} \delta_{k_{n}}^{k_{\alpha_{3}(n)}} T_{i_{1} j_{1} k_{1}} \cdots T_{i_{n-1} j_{n-1} k_{n-1}} \bar{T}^{i_{\alpha_{1}(1)} j_{\alpha_{2}(1)} k_{\alpha_{3}(1)}} \cdots \bar{T}^{i_{\alpha_{1}(n-1)} j_{\alpha_{2}(n-1)}} k_{\alpha_{3}(n-1)}=N^{a} \mathcal{O}_{\vec{\alpha}}
$$

where $a$ is the number of $\alpha$ 's for which $\alpha_{i}(n)=n$. This is implemented in Eq. (E6) as

$$
\Delta \mathcal{O}_{\vec{\mu}}=\frac{n^{3}}{n !^{d}} \frac{g_{\vec{\mu}}}{g_{\vec{\mu}} \uparrow \vec{\mu}} \sum_{\vec{\alpha}^{\prime}, \sigma^{\prime}} d_{\vec{\mu}} \prod_{k=1}^{3}\left[N \chi_{\mu_{k}}\left(\alpha_{k}^{\prime} \sigma^{\prime}\right)+\chi_{\mu_{k}}\left(\mathcal{J}_{n} \alpha_{k}^{\prime} \sigma^{\prime}\right)\right] \mathcal{O}_{\alpha^{\prime}}
$$

Applying Eq. (C11) and the fact that

$$
\chi_{\mu}\left(\alpha^{\prime}\right)=\sum_{\mu^{\prime} \nearrow_{\mu}} \chi_{\mu^{\prime}}\left(\alpha^{\prime}\right)
$$

we can write

$$
\begin{aligned}
\Delta \mathcal{O}_{\vec{\mu}} & =\frac{n^{3}}{n !^{d}} \frac{g_{\vec{\mu}}}{g_{\vec{\mu}^{\prime} \uparrow \vec{\mu}}} \sum_{\vec{\alpha}^{\prime}, \sigma^{\prime}} \sum_{\vec{\mu}^{\prime} \nearrow \vec{\mu}} d_{\vec{\mu}} \chi_{\vec{\mu}^{\prime}}\left(\vec{\alpha}^{\prime} \sigma^{\prime}\right) \prod_{k=1}^{3}\left[N+J\left(\mu_{k}, \mu_{k}^{\prime}\right)\right] \mathcal{O}_{\vec{\alpha}^{\prime}} \\
& =\frac{n^{3}(n-1) !^{d}}{n !^{d}} \frac{g_{\vec{\mu}}}{g_{\vec{\mu}^{\prime} \uparrow \vec{\mu}}} \sum_{\vec{\mu}^{\prime} \nearrow \vec{\mu}} \frac{d_{\vec{\mu}}}{d_{\vec{\mu}^{\prime}}} \prod_{k=1}^{3}\left[N+J\left(\mu_{k}, \mu_{k}^{\prime}\right)\right] \mathcal{O}_{\vec{\mu}^{\prime}} \\
& =n^{3} \frac{g_{\vec{\mu}}}{g_{\vec{\mu}^{\prime} \uparrow \vec{\mu}}} \sum_{\vec{\mu}^{\prime} \nearrow \vec{\mu}} \frac{\operatorname{Dim}_{\vec{\mu}}(N)}{\operatorname{Dim}_{\vec{\mu}^{\prime}}(N)} \mathcal{O}_{\vec{\mu}^{\prime}},
\end{aligned}
$$

where, on the last line of Eq. (E10), we have applied

$$
N+J\left(\mu_{k}, \mu_{k}^{\prime}\right)=\frac{f_{\mu_{k}}}{f_{\mu_{k}^{\prime}}}=n \frac{\operatorname{Dim}_{\vec{\mu}}(N)}{\operatorname{Dim}_{\vec{\mu}^{\prime}}(N)} \frac{d_{\mu_{k}^{\prime}}}{d_{\mu_{k}}} .
$$

Note that the factor $n^{3}$ on the last line of Eq. (E10) is general and valid for a tensor of any order. The result (E10) is proof that the cut operator is closed when acting on Schur invariants $\mathcal{O}_{\vec{\mu}}$. 
[1] R. De Mello Koch, D. Gossman, N. H. Tahiridimbisoa, and A. L. Mahu, Holography for tensor models, Phys. Rev. D 101, 046004 (2020).

[2] P. Di Francesco, P. H. Ginsparg, and J. Zinn-Justin, 2-D gravity and random matrices, Phys. Rep. 254, 1 (1995).

[3] J. Ambjorn, B. Durhuus, and T. Jonsson, Three-dimensional simplicial quantum gravity and generalized matrix models, Mod. Phys. Lett. A 06, 1133 (1991).

[4] M. Gross, Tensor models and simplicial quantum gravity in 2-D, Nucl. Phys. B, Proc. Suppl. 25, 144 (1992).

[5] N. Sasakura, Tensor model for gravity and orientability of manifold, Mod. Phys. Lett. A 06, 2613 (1991).

[6] R. Gurau, Colored group field theory, Commun. Math. Phys. 304, 69 (2011).

[7] R. Gurau and J. P. Ryan, Colored tensor models-A review, SIGMA 8, 020 (2012).

[8] R. Gurau, The 1/N expansion of colored tensor models, Ann. Henri Poincaré 12, 829 (2011).

[9] R. Gurau and V. Rivasseau, The $1 / \mathrm{N}$ expansion of colored tensor models in arbitrary dimension, Europhys. Lett. 95, 50004 (2011).

[10] R. Gurau, The complete $1 / \mathrm{N}$ expansion of colored tensor models in arbitrary dimension, Ann. Henri Poincaré 13, 399 (2012).

[11] J. B. Geloun and S. Ramgoolam, Counting tensor model observables and branched covers of the 2-sphere, Ann. Inst. Henri Poincaré D 1, 77 (2014).

[12] P. Diaz and S. J. Rey, Orthogonal bases of invariants in tensor models, J. High Energy Phys. 02 (2018) 089.

[13] J. Ben Geloun and S. Ramgoolam, Tensor models, Kronecker coefficients and permutation centralizer algebras, J. High Energy Phys. 11 (2017) 092.

[14] H. Itoyama, A. Mironov, and A. Morozov, Cut and join operator ring in Aristotelian tensor model, Nucl. Phys. B932, 52 (2018).

[15] P. Diaz and S. J. Rey, Invariant operators, orthogonal bases and correlators in general tensor models, Nucl. Phys. B932, 254 (2018).

[16] P. Diaz, Tensor and matrix models: A one-night stand or a lifetime romance?, J. High Energy Phys. 06 (2018) 140.

[17] P. Diaz and J. A. Rosabal, Spontaneous symmetry breaking in tensor theories, J. High Energy Phys. 01 (2019) 094.

[18] H. Itoyama, A. Mironov, and A. Morozov, Complete solution to Gaussian tensor model and its integrable properties, Phys. Lett. B 802, 135237 (2020).

[19] G. Kemp and S. Ramgoolam, BPS states, conserved charges and centres of symmetric group algebras, J. High Energy Phys. 01 (2020) 146.

[20] R. M. Koch, D. Gossman, and L. Tribelhorn, Gauge invariants, correlators and holography in bosonic and fermionic tensor models, J. High Energy Phys. 09 (2017) 011.

[21] H. Itoyama, A. Mironov, and A. Morozov, From Kronecker to tableau pseudo-characters in tensor models, Phys. Lett. B 788, 76 (2019).

[22] N. Delporte and V. Rivasseau, The tensor track V: Holographic tensors, arXiv:1804.11101.

[23] I. R. Klebanov, F. Popov, and G. Tarnopolsky, TASI lectures on large $N$ tensor models, Proc. Sci., TASI2017 (2018) 004 [arXiv:1808.09434].
[24] V. Bonzom, R. Gurau, A. Riello, and V. Rivasseau, Critical behavior of colored tensor models in the large $N$ limit, Nucl. Phys. B853, 174 (2011).

[25] A. M. Vershik and S. V. Kerov, Asymptotic character theory of the symmetric group, Funct. Anal. Appl. 15, 246 (1981).

[26] E. Thoma, Die unzerlegbaren, positiv-definiten Klassenfunktionen der abzählbar unendlichen, symmetrischen Gruppe, Math. Z. 85, 40 (1964).

[27] A. Okounkov, On representations of the infinite symmetric group, J. Math. Sci. 96, 3550 (1999).

[28] P. Diaz, The non-melonic sector of tensor models and gravity, arXiv:1912.00208.

[29] V. Balasubramanian, M. Berkooz, A. Naqvi, and M. J. Strassler, Giant gravitons in conformal field theory, J. High Energy Phys. 04 (2002) 034.

[30] R. C. Avohou, J. B. Geloun, and N. Dub, On the counting of $O(N)$ tensor invariants, Adv. Theor. Math. Phys. 24, 821 (2020).

[31] J. B. Geloun, On the counting tensor model observables as $U(N)$ and $O(N)$ classical invariants, Proc. Sci., CORFU2019 (2020) 175 [arXiv:2005.01773].

[32] S. Corley, A. Jevicki, and S. Ramgoolam, Exact correlators of giant gravitons from dual $N=4$ SYM theory, Adv. Theor. Math. Phys. 5, 809 (2001).

[33] R. Bhattacharyya, S. Collins, and R. de Mello Koch, Exact multi-matrix correlators, J. High Energy Phys. 03 (2008) 044.

[34] R. Bhattacharyya, R. de Mello Koch, and M. Stephanou, Exact multi-restricted Schur polynomial correlators, J. High Energy Phys. 06 (2008) 101.

[35] Y. Kimura and S. Ramgoolam, Branes, anti-branes and Brauer algebras in gauge-gravity duality, J. High Energy Phys. 11 (2007) 078.

[36] T. W. Brown, P. J. Heslop, and S. Ramgoolam, Diagonal multi-matrix correlators and BPS operators in $N=4$ SYM, J. High Energy Phys. 02 (2008) 030 .

[37] P. Mattioli and S. Ramgoolam, Permutation centralizer algebras and multi-matrix invariants, Phys. Rev. D 93, 065040 (2016).

[38] R. Gurau, Universality for random tensors, Ann. Inst. Henri Poincaré Probab. Stat. 50, 1474 (2014).

[39] A. Eichhorn, J. Lumma, A. D. Pereira, and A. Sikandar, Universal critical behavior in tensor models for fourdimensional quantum gravity, J. High Energy Phys. 02 (2020) 110.

[40] P. Biane, Representations of symmetric groups and free probability, Adv. Math. 138, 126 (1998).

[41] A. Borodin and G. Olshanski, The Young bouquet and its boundary, Moscow Math. J. 13, 193 (2013).

[42] I. Pak, G. Panova, and D. Yeliussizov, On the largest Kronecker and Littlewood-Richardson coefficients, J. Comb. Theory Ser. A 165, 44 (2019).

[43] T. Biswas, E. Gerwick, T. Koivisto, and A. Mazumdar, Towards Singularity and Ghost Free Theories of Gravity, Phys. Rev. Lett. 108, 031101 (2012).

[44] H. Itoyama, A. Mironov, and A. Morozov, Ward identities and combinatorics of rainbow tensor models, J. High Energy Phys. 06 (2017) 115. 
[45] H. Itoyama and R. Yoshioka, Generalized cut operation associated with higher order variation in tensor models, Nucl. Phys. B945, 114681 (2019).

[46] R. de Mello Koch, Geometries from Young diagrams, J. High Energy Phys. 11 (2008) 061.
[47] R. de Mello Koch, J.H. Huang, and L. Tribelhorn, Exciting LLM geometries, J. High Energy Phys. 07 (2018) 146.

[48] P. Diaz (to be published). 